ARTICLE

Received 13 Jan 2014 | Accepted 31 Jul 2014 | Published 11 Sep 2014 DOI: 10.1038/ncomms5857

\title{
Mechanistic insight into GPCR-mediated activation of the microtubule-associated RhoA exchange factor GEF-H1
}

David Meiri ${ }^{1, *}$, Christopher B. Marshall ${ }^{2, \star}$, Daphna Mokady², Jose LaRose ${ }^{2}$, Michael Mullin $^{3}$, Anne-Claude Gingras ${ }^{3,4}$, Mitsuhiko Ikura ${ }^{2,5}$ \& Robert Rottapel 2,5,6,7,8

The RhoGEF GEF-H1 can be sequestered in an inactive state on polymerized microtubules by the dynein motor light-chain Tctex-1. Phosphorylation of GEF-H1 Ser885 by PKA or PAK kinases creates an inhibitory 14-3-3-binding site. Here we show a new mode of GEF-H1 activation in response to the G-protein-coupled receptor (GPCR) ligands lysophosphatidic acid (LPA) or thrombin that is independent of microtubule depolymerization. LPA/thrombin stimulates disassembly of the GEF-H1:dynein multi-protein complex through the concerted action of $\mathrm{G} \alpha$ and $\mathrm{G} \beta \gamma$. $\mathrm{G} \alpha$ binds directly to GEF-H1 and displaces it from Tctex-1, while $\mathrm{G} \beta \gamma$ binds to Tctex-1 and disrupts its interaction with the dynein intermediate chain, resulting in the release of GEF-H1. Full activation of GEF-H1 requires dephosphorylation of Ser885 by PP2A, which is induced by thrombin. The coordinated displacement of GEF-H1 from microtubules by G-proteins and its dephosphorylation by PP2A demonstrate a multistep GEF-H1 activation and present a unique mechanism coupling GPCR signalling to Rho activation.

\footnotetext{
${ }^{1}$ Department of Biology, Technion Israel Institute of Technology, Haifa 32000, Israel. ${ }^{2}$ Princess Margaret Cancer Centre, University Health Network, University of Toronto, 101 College Street, Room 12-704, Toronto Medical Discovery Tower, Toronto, Ontario, Canada M5G 1L7. ${ }^{3}$ Lunenfeld-Tanenbaum Research Institute, Mount Sinai Hospital, 600 University Avenue, Room 992A, Toronto, Ontario, Canada M5G 1X5. ${ }^{4}$ Department of Molecular Genetics, University of Toronto, Toronto, Ontario, Canada M5S 1A8. ${ }^{5}$ Department of Medical Biophysics, University of Toronto, 1 King's College Circle, Toronto, Ontario, Canada M5S 1A8. ${ }^{6}$ Department of Medicine, University of Toronto, 1 King's College Circle, Toronto, Ontario, Canada M5S 1A8. ${ }^{7}$ Department of Immunology, University of Toronto, 1 King's College Circle, Toronto, Ontario, Canada M5S 1A8. ${ }^{8}$ Division of Rheumatology, St Michael's Hospital, 30 Bond Street, Toronto, Ontario, Canada M5B 1W8. * These authors contributed equally to this work. Correspondence and requests for materials should be addressed to R.R. (email: rottapel@uhnresearch.ca).
} 
$\mathrm{T}$ he Rho GTPases are critical modulators of cell morphology and motility through their regulation of the actin cytoskeleton. The activation state of Rho GTPases is governed by the balance between the activities of GTPaseactivating proteins, which catalyse hydrolysis of guanosine- $5^{\prime}$ triphosphate (GTP) and guanine exchange factors (GEFs), which promote GTP binding. GEF-H1, also known as ARHGEF2 or Lfc in mouse, is associated with microtubules in its inactive state $^{1}$. Expression of GEF-H1 promotes stress fibre formation through RhoA activation, while its depletion attenuates actin reorganization ${ }^{2-5}$. A mutant of GEF-H1 unable to bind to microtubules has increased GEF activity and induces stress fibre formation $^{5}$, suggesting that GEF-H1 is a critical factor mediating crosstalk between microtubules and actin ${ }^{5}$. We have shown that the dynein light-chain Tctex-1 tethers GEF-H1 to polymerized microtubules $^{6}$, where it is poised to undergo D-AKAP-1dependent phosphorylation of Ser885 by PKA. Phosphorylation of Ser885 creates a high-affinity 14-3-3-binding site that inhibits the exchange activity of GEF-H1 (ref. 3). Nocodazole-induced depolymerization of microtubules disrupts this inhibited GEF-H1 complex resulting in potent activation of GEF-H1, suggesting that cycles of microtubule depolymerization under physiologic conditions would release GEF-H1, providing local and temporally constrained activation of RhoA ${ }^{6,7}$.

We and others have shown that a variety of receptor ligands including lysophosphatidic acid (LPA) ${ }^{3}$, thrombin ${ }^{2}$, tumour necrosis factor- $\alpha$ and epidermal growth factor ${ }^{8,9}$ lead to activation of GEF-H1 through unknown mechanisms. Moreover, GEF-H1 can be activated by mechanotransduction through an integrin-mediated mechanism, which is suggested to be independent of microtubule depolymerization ${ }^{10}$.

In the present study, we sought to determine the mechanism underlying GEF-H1 activation in response to the GPCR (Gprotein-coupled receptor) ligands LPA and thrombin. $\mathrm{G} \alpha_{12 / 13}$ proteins play a well-established role in the activation of RhoGEFs belonging to the regulator of G-protein signalling (RGS) domaincontaining RhoGEF subfamily, which includes p115-RhoGEF, PDZ-RhoGEF, leukemia-associated RhoGEF (LARG) and possibly Lbc-RhoGEF ${ }^{11-13}$. $G \alpha_{12 / 13}$ subunits bind directly to the RGShomology $(\mathrm{RH})$ domain contained within the polypeptide sequences of these GEFs, which is thought to alleviate autoinhibition of their exchange activity. Although GEF-H1 is known to respond to $\mathrm{G} \alpha_{12 / 13}$-coupled GPCR stimulation, to date no interaction with $G \alpha_{12 / 13}$ has been shown, nor has an $R H$ domain been identified in its polypeptide sequence.

Here we identify a novel mechanism for GEF-H1 activation mediated by the coincident interaction of $\mathrm{G} \alpha_{12 / 13}$ with GEF-H1 and G $\beta \gamma$ with Tctex-1, resulting in the release and activation of GEF-H1 from the inhibitory dynein-associated complex. In addition, we show that thrombin stimulates the interaction between PP2A and GEF-H1 triggering dephosphorylation of Ser885, which is required for activation. This study provides a detailed multistep mechanism for GPCR-mediated activation of GEF-H1 independent of microtubule depolymerization.

\section{Results}

LPA/thrombin induce GEF-H1 release from intact microtubules. GEF-H1 is required for LPA/thrombin-induced stress fibre formation, and focal adhesion formation following stimulation with tumour necrosis factor- $\alpha$, LPA or thrombin ${ }^{2,3,6}$. We have recently shown that microtubule-dependent inhibition of GEF-H1 requires the assembly of a protein complex including Tctex-1, dynein intermediate chain (DIC) and 14-3-3 proteins ${ }^{6}$. We examined whether the microtubule-associated inhibited GEF-H1 complex is altered following LPA or thrombin stimulation, and observed that $5 \mathrm{~min}$ following treatment with LPA or thrombin, the stoichiometry of DIC, 14-3-3 and Tctex-1 present in GEF-H1 immune complexes markedly decreased compared with basal conditions (Fig. 1a).

Dissolution of the interaction between DIC-Tctex-1 and GEFH1 might represent one mechanism by which growth factor stimulation could activate GEF-H1 by displacement from the microtubule array. We investigated whether LPA or thrombin stimulation alters GEF-H1 localization. GEF-H1-knockout murine embryonic fibroblasts (MEFs) were transfected with GEF-H1enhanced green fluorescent protein (eGFP) and visualized by confocal microscopy before and after treatment. In untreated cells, GEF-H1-eGFP was closely associated with the microtubule array (correlation coefficient of $0.67, n=91$ ), whereas after $5 \mathrm{~min}$ of LPA or thrombin treatment GEF-H1-eGFP moved into a largely cytoplasmic distribution (Fig. 1b-d). Similar to GEF-H1 localization after application of force ${ }^{10}$, we noted that a fraction of GEF-H1 relocalized to vinculin-positive focal adhesion structures following treatment with LPA (correlation coefficient of $0.81, n=74$ ) (Fig. 1e; Supplementary Fig. 1). Importantly, acute treatment with LPA or thrombin did not depolymerize the microtubule network, suggesting that under these conditions GEF-H1 displacement was not contingent on disruption of microtubule arrays (Fig. 1c). To address the possibility that growth factor stimulation of cells increased microtubule dynamics, which may not be observable under steady-state conditions, we visualized live cells expressing cherry tubulin and GEF-H1-eGFP using real-time confocal microscopy. Live imaging verified that the bulk of the microtubule array remained intact during the time interval of the stimulation coincident with GEF-H1 relocalization into the cytoplasm (Fig. 1f).

The G $\beta$ subunit binds to Tctex-1 and activates GEF-H1. LPA and thrombin signal to cells through activation of the LPA receptors and protease-activated GPCRs, respectively. Inactive GPCRs associate with $\alpha$-, $\beta$ - and $\gamma$-subunits (known as $G \alpha, G \beta$ and $G \gamma$, respectively), however, upon ligand activation, the $G \alpha$ subunit dissociates from the $G \beta \gamma$ heterodimer, where upon both subunits can engage multiple intracellular signalling pathways.

Sachdev et al. ${ }^{15}$ showed that the G $\beta$ subunit binds to Tctex-1 and competes with DIC for binding to Tctex-1, regulating assembly of Tctex-1 into the dynein motor complex ${ }^{14,15}$. We asked whether G $\beta \gamma$ subunits might impinge on the GEFH1:Tctex-1:dynein complex, leading to the displacement of GEF-H1 from microtubules and its activation. We confirmed that G $\beta$ bound to Tctex-1 in 293T cells co-expressing $G \beta_{12}$ isoform, Tctex-1 and GEF-H1, while a G $\beta$ variant $\left(\mathrm{G} \beta_{\text {mut }}\right)$ bearing three mutations (R48A, R49A and R52A) in the Tctex-1binding site pulled down significantly less Tctex-1 (Fig. 2a), consistent with results reported by Sachdev et al. ${ }^{15}$ To determine whether G $\beta$ binding to Tctex-1 disrupts the interaction between GEF-H1 and Tctex-1, GEF-H1 immune complexes were analysed for the presence of Tctex-1 in the absence or presence of the expressed G $\beta_{12}$ subunit. While Tctex-1 was detected in GEF-H1 immunoprecipitates, expression of $\mathrm{G} \beta$ markedly reduced the association of GEF-H1 with Tctex-1 protein (Fig. 2b). The specificity of this effect was demonstrated by the observation that $G \beta_{\text {mut }}$ expression did not alter the GEF-H1:Tctex-1 interaction (Fig. 2b). These data suggest that the G $\beta$ subunit can disrupt the anchoring function of Tctex-1 that links GEF-H1 to microtubules. We therefore examined whether $G \beta_{12} \gamma_{12}$ expression could alter GEF-H1 localization to the microtubule array using fluorescence microscopy. We observed that the localization of GEF-H1-eGFP, normally intimately associated 


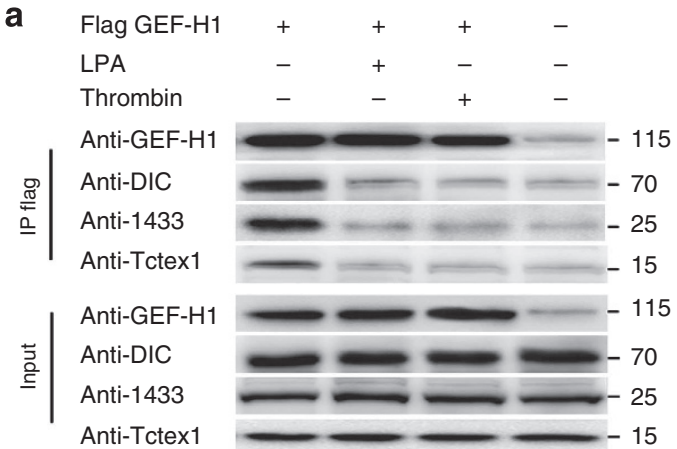

b
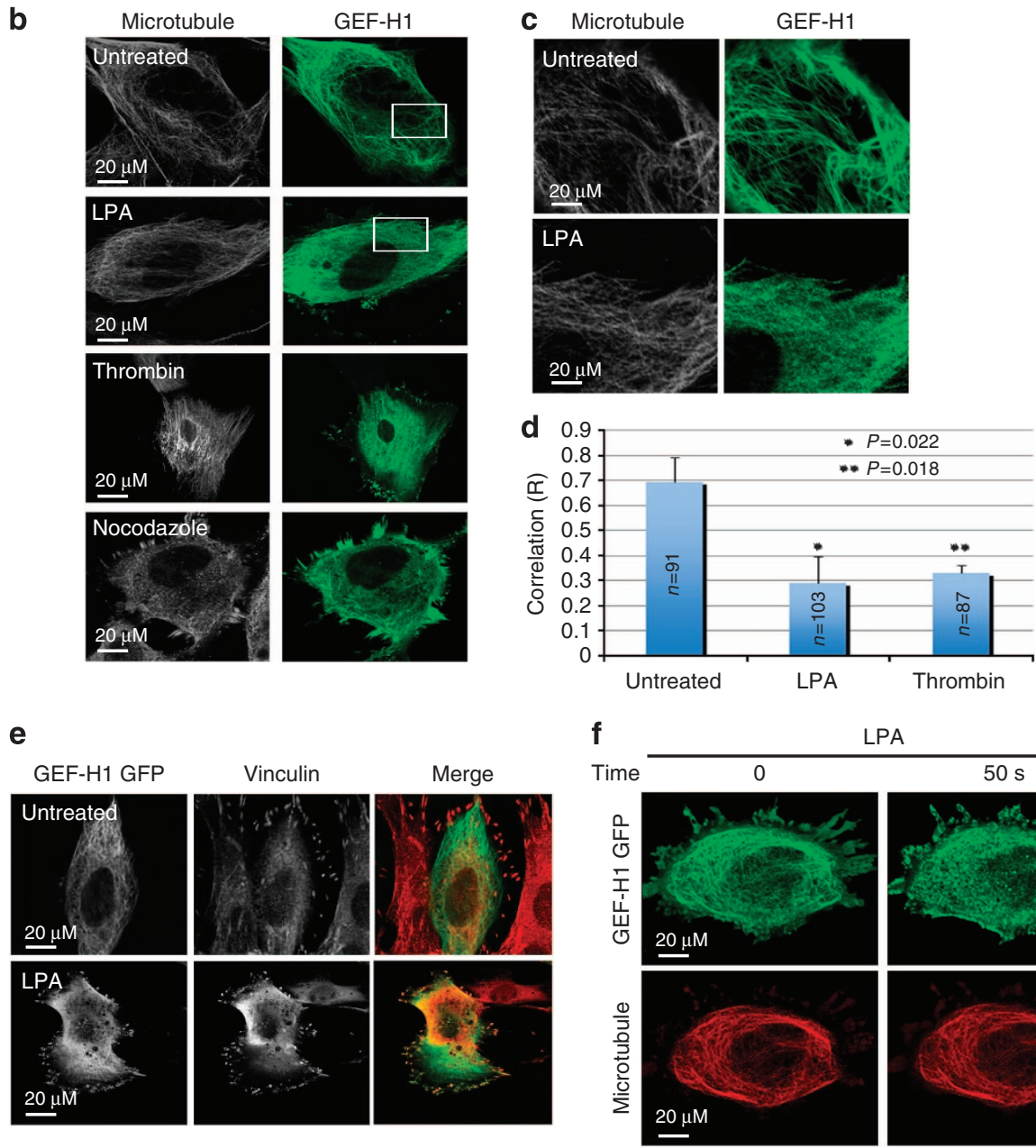

LPA
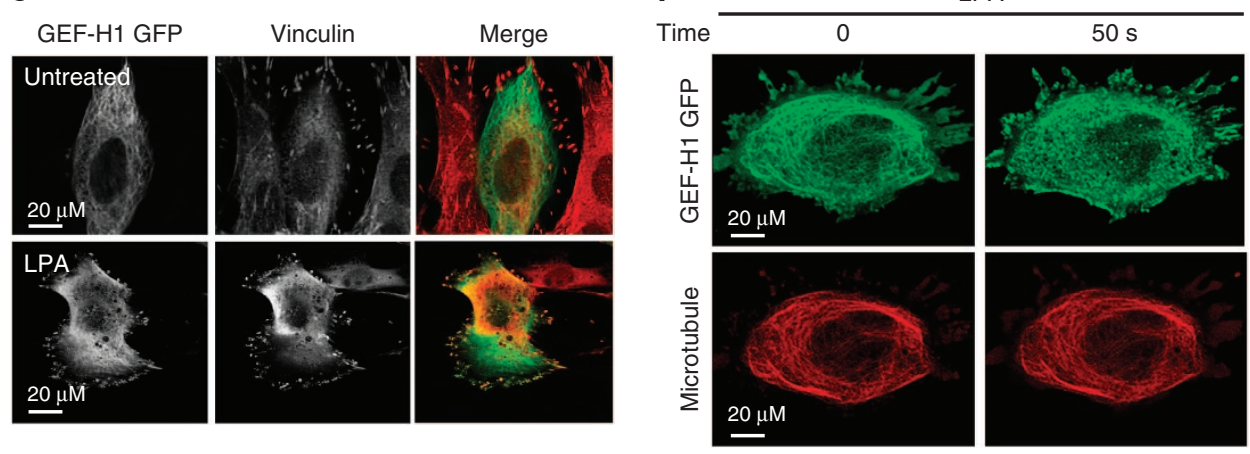

Figure 1 | GEF-H1 translocates from microtubules to the cytoplasm and focal adhesions in response to LPA or thrombin. (a) LPA or thrombin treatment disrupts GEF-H1-dynein complex. Serum-starved HEK293T cells, expressing Flag-tagged GEF-H1, were treated with LPA or thrombin for 5 min. Endogenous DIC, 14-3-3 and Tctex-1 proteins were detected in Flag-GEF-H1-precipitated complexes by western blot. Western blots of the whole-cell lysate (input) and immunoprecipitated complexes (IP) are shown. (b) LPA or thrombin induced GEF-H1 relocalization from microtubules. Confocal images of GEF-H1 -/- MEFs transfected with GEF-H1-eGFP were treated with LPA, thrombin or nocodazole, as indicated, for 5 min prior to fixation and staining with anti-tubulin. Scale bar, $20 \mu \mathrm{m}$. (c) Higher magnification $(5 \times 60)$ view of the images depicted in $\mathbf{b}$. Scale bar, $20 \mu \mathrm{m}$. (d) The correlation coefficient $(R)$ measuring co-localization between eGFP (GEF-H1) and polymerized microtubules in $\mathbf{b}$ was determined in 90 cells from three independent experiments. Error bars represent s.d. of at least three independent replicates, and $P$ values derived from pairwise $t$-tests are indicated. (e) GEF-H1 translocates to focal adhesions in response to LPA. GEF-H1 ${ }^{-/-}$MEFs overexpressing GEF-H1-eGFP were treated with LPA for $1 \mathrm{~h}$ prior to fixation and staining with anti-vinculin antibodies (red) to visualize focal adhesions. Co-localization between GEF-H1 (GFP) and vinculin was measured with and without LPA treatment. Scale bar, $20 \mu \mathrm{m}$. (f) GEF-H1 relocalization using live-cell imaging. Images from movies of GEF-H1 ${ }^{-/}-\mathrm{MEF}$ cells transiently transfected with GEF-H1-eGFP and cherry tubulin, before and after LPA stimulation. Starved cells were treated with LPA. Data are representative of three independent biological replicates. Scale bar, $20 \mu \mathrm{m}$. 
a

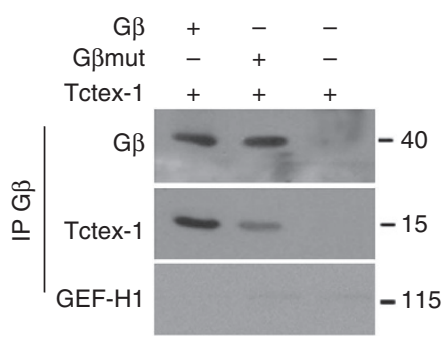

b

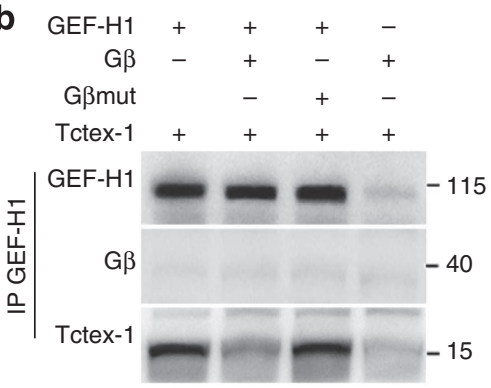

C

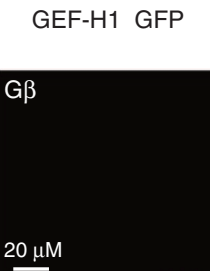

GEF-H1 GFP+

GEF-H1 GFP+ $\mathrm{G} \beta \gamma$

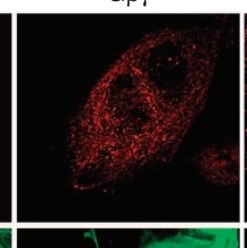
G $\beta \gamma+$ bARKct
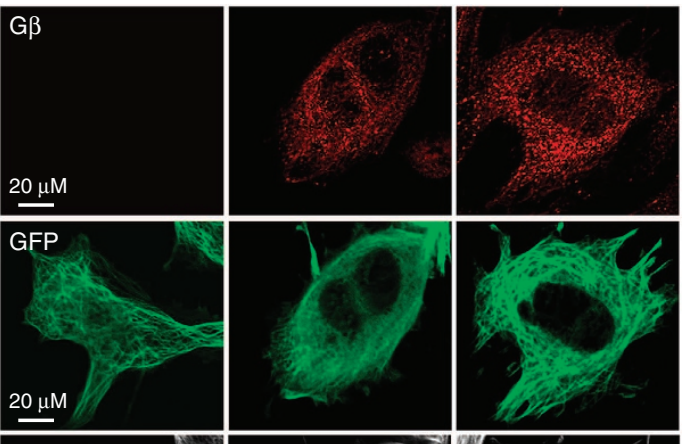

d
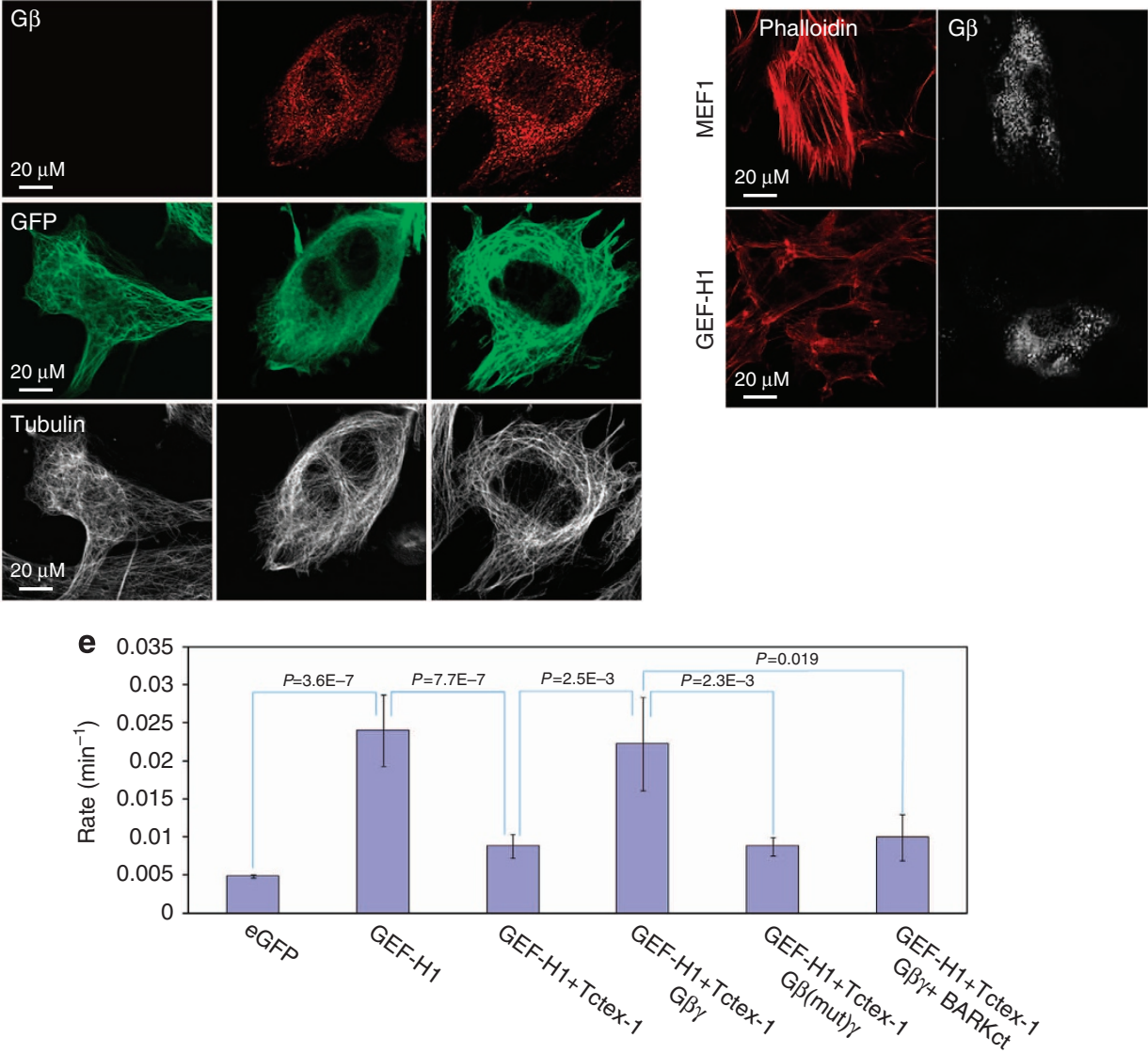

Figure 2 | G $\beta$ subunit binds to Tctex-1 and activates GEF-H1. (a) G $\beta$ subunit binds Tctex-1 in HEK293T cells. Flag-Tctex-1 was expressed in HEK293T cells alone or co-expressed with $G \beta$ or a $G \beta$ variant with mutations at the Tctex-1-binding site (G $\beta_{\text {mut, }}$ R48A, R49A and R52A). Cells were lysed and G $\beta$ protein complexes precipitated with anti-G $\beta$ antibody were probed with anti-G $\beta$, anti-Tctex-1 and anti-GEF-H1 antibodies. (b) G $\beta$ displaces GEF-H1 from Tctex-1. Myc-GEF-H1 and Flag-Tctex-1 were expressed with $\mathrm{G} \beta_{12}$ or $\mathrm{G} \beta_{\text {mut }}$ in HEK293T cells, and following lysis, GEF-H1 complexes were immunoprecipitated with anti-Myc antibody, and probed with anti-G $\beta$, anti-Tctex-1 or anti-GEF-H1 antibodies, respectively. (c) G $\beta_{12} \gamma_{12}$ overexpresion induces GEF-H1 relocalization. Confocal images of GEF-H1 - / - cells expressing GEF-H1-eGFP or co-expressing GEF-H1-eGFP and G $\beta_{12} \gamma_{12}$, with or without $\beta A R K c t$ ( $G \beta \gamma$-specific antagonist). Cells were fixed 1 day after transfection and stained with anti-G $\beta$ or $\alpha$-tubulin antibodies. Scale bar, $20 \mu$ m. (d) $G \beta_{12} \gamma_{12}$ expression induces stress fibre formation in a GEF-H1-dependent manner. Wild-type or GEF-H1 $-/-M E F s$, expressing G $\beta_{12} \gamma_{12}$ proteins, were fixed 1 day after transfection, stained with anti-G $\beta$ and anti-phalloidin antibodies and visualized by confocal microscopy. Scale bar, $20 \mu \mathrm{m}$. (e) G $\beta \gamma$ expression activates GEF-H1 exchange activity in a specific manner. RhoA nucleotide exchange rates in the presence of lysates derived from HEK293T cells overexpressing eGFP alone, GEF-H1-eGFP or co-expressing GEF-H1-eGFP and Flag-Tctex-1 with or without co-transfection of G $\beta \gamma$, G $\beta_{\text {mut }} \gamma$ or G $\beta \gamma$ and the inhibitory $\beta$ ARKct peptide. GEF-H1, Tctex-1 and $\mathrm{G} \beta$ protein levels in cell lysates were detected by western blots. Error bars represent s.d. of at least three independent replicates, and $P$ values derived from pairwise $t$-tests are indicated.

with the microtubule array, redistributed into a diffuse pattern

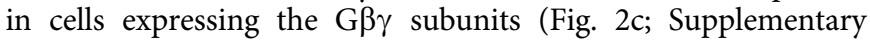

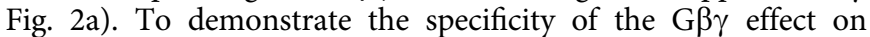
GEF-H1 redistribution, we co-expressed the G $\beta \gamma$-specific antagonist peptide, $\beta$ ARKct, derived from the G $\beta \gamma$-binding domain of $\beta$-adrenergic receptor kinase 1 , which specifically inhibits $G \beta \gamma$-dependent signalling ${ }^{16}$, and noted that coexpression of $\beta$ ARKct restored GEF-H1-eGFP localization to the microtubules (Fig. 2c; Supplementary Fig. 2a). We next queried whether $G \beta \gamma$ expression could induce stress fibre formation in a GEF-H1-dependent manner following the redistribution of GEF-H1 from the microtubule array. We 
found that expression of the $G \beta \gamma$ subunits was sufficient to induce actin-based stress fibres in wild-type MEFs but not in GEF-H1 knockout MEFs (Fig. 2d), reinforcing the idea that G $\beta \gamma$ plays a role in inducing stress fibre formation through GEF-H1. Last, we investigated whether expression of $G \beta \gamma$ could regulate the catalytic activity of GEF-H1. As previously reported, Tctex-1 potently inhibited the exchange activity of full-length GEF-H1 (refs 6,17), whereas co-expression of the G $\beta \gamma$ subunits relieved this inhibitory effect (Fig. 2e). In contrast, expression of the $G \beta_{\text {mut }}$ peptide was unable to antagonize the inhibitory effect of Tctex-1 (Fig. 2e; Supplementary Fig. 2b). Co-expression of the $\beta A R K c t$ inhibitory peptide blocked the ability of $G \beta \gamma$ to antagonize Tctex-1-mediated inhibition of GEF-H1 (Fig. 2e; Supplementary Fig. 2b), demonstrating the specificity of the G $\beta \gamma$ effect. These data demonstrate that G $\beta \gamma$ can disrupt the interaction of GEF-H1 with the dynein motor complex leading to its displacement from the microtubule array and activation of its exchange activity.

$\mathrm{G} \alpha_{12 / 13}$ subunit induces GEF-H1 localization and activation. $\mathrm{G} \alpha_{12 / 13}$ subunits directly activate a class of RhoGEFs including p115-RhoGEF, PDZ-RhoGEF, LARG and Lbc-RhoGEF ${ }^{1,12}$ that contain $\mathrm{G} \alpha$-binding $\mathrm{RH}$ domains. LPA or thrombin signalling through $\mathrm{G} \alpha_{12 / 13}$ subunits induces stress fibres in a manner that requires GEF-H1 (ref. 5), yet GEF-H1 does not have an identifiable RGS domain. We directly tested whether the induction of stress fibres by $G \alpha_{12}$ and $G \alpha_{13}$ was dependent on GEF-H1. We observed that wild-type, but not GEF-H1-knockout, MEFs expressing $\mathrm{G} \alpha_{12}$ or $\mathrm{G} \alpha_{13}$ subunits contained high levels of polymerized actin (Fig. 3a; Supplementary Fig. 3). The requirement of GEF-H1 for stress fibre formation suggested that this aspect of $G \alpha_{12 / 13}$ signalling operates through the GEFH1 pathway. We next examined whether constitutively active $\mathrm{G} \alpha_{12}$ or $\mathrm{G} \alpha_{13}$ could trigger a change in GEF-H1 localization and stimulate GEF-H1 activation. GEF-H1 ${ }^{-/}$cells were transfected with GEF-H1-eGFP alone or together with a constitutively active $\mathrm{G} \alpha_{12 / 13}$ mutant $\left(\mathrm{G} \alpha_{12 \mathrm{QL}}, \mathrm{G} \alpha_{13 \mathrm{QL}}\right)$, fixed and stained with phalloidin or anti-tubulin antibodies. GEF-H1-eGFP localized to microtubules in starved cells that contained little polymerized actin (Fig. 3b,c; Supplementary Fig. 3). Expression of $\mathrm{G} \alpha_{12 \mathrm{QL}}$ or $\mathrm{G} \alpha_{13 \mathrm{QL}}$ induced the redistribution of GEF-H1-eGFP from the microtubule array to a diffuse cytoplasmic pattern and was associated with stress fibre formation (Fig. 3b,c; Supplementary Fig. 3), both of which were blocked by the co-expression of the RGS domain of p115 (rgRGS), which specifically binds and blocks $\mathrm{G} \alpha_{12 / 13}$ (refs 18,19). This demonstrates that the $\mathrm{RH}$ domainbinding surface of $\mathrm{G}_{12 \mathrm{QL}}$ is required to regulate GEF-H1 redistribution from the microtubules.

The G $\alpha$ subunit binds directly to GEF-H1. To determine whether activation of GEF-H1 by $\mathrm{G} \alpha_{12 / 13}$ is mediated by a direct binding event, we performed pull-down experiments with purified recombinant $\mathrm{G}_{13}$ (a chimera of $\mathrm{G}_{\mathrm{i} 1}$ residues 1-28 fused to $\mathrm{G} \alpha_{13}$ residues $41-377$ (ref. 20)) and GEF-H1 proteins. Recombinant full-length GEF-H1 protein expressed and purified from insect cells ${ }^{6}$ bound to recombinant $G \alpha_{13}$, indicating that active $\mathrm{G} \alpha$ can bind directly to GEF-H1 in vitro (Supplementary Fig. 4a). A series of GEF-H1 truncations and deletions revealed that the $\mathrm{G} \alpha_{12 / 13}$-binding site resides in the $\mathrm{N}$-terminal region of GEF-H1 (Fig. 4a; Supplementary Fig. 4b). Coimmunoprecipitation experiments with a series of Flag-tagged, $\mathrm{N}$-terminal expressed GEF-H1 fragments demonstrated that amino acids 1-240 of GEF-H1 are sufficient for optimal binding to endogenous $G \alpha_{12}$ while shorter deletions within this region markedly reduced $\mathrm{G} \alpha$ binding (Fig. $4 \mathrm{~b}$ ). The requirement

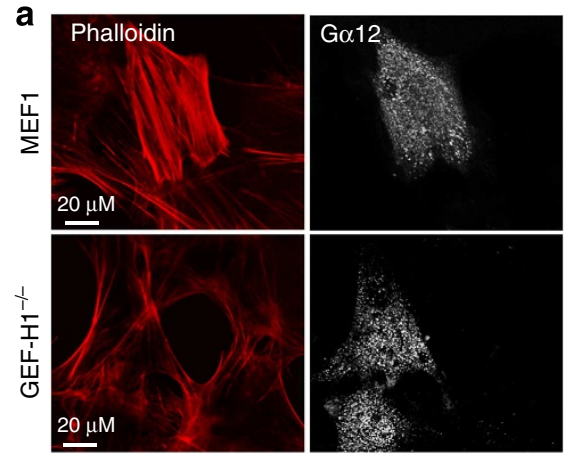

b
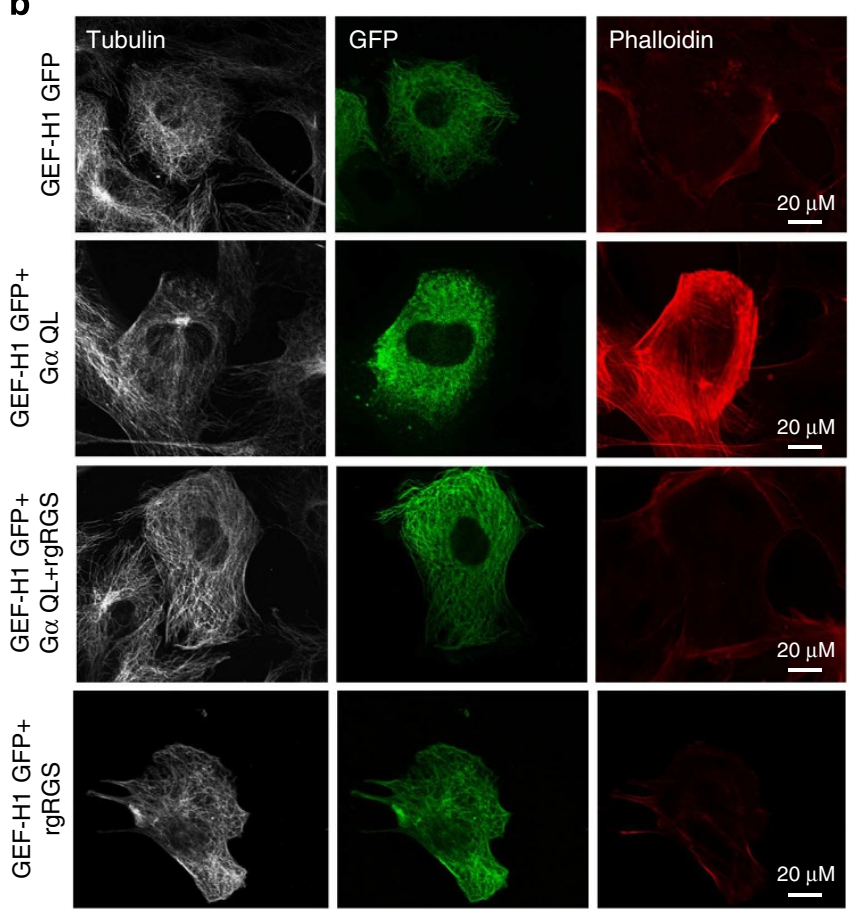

C
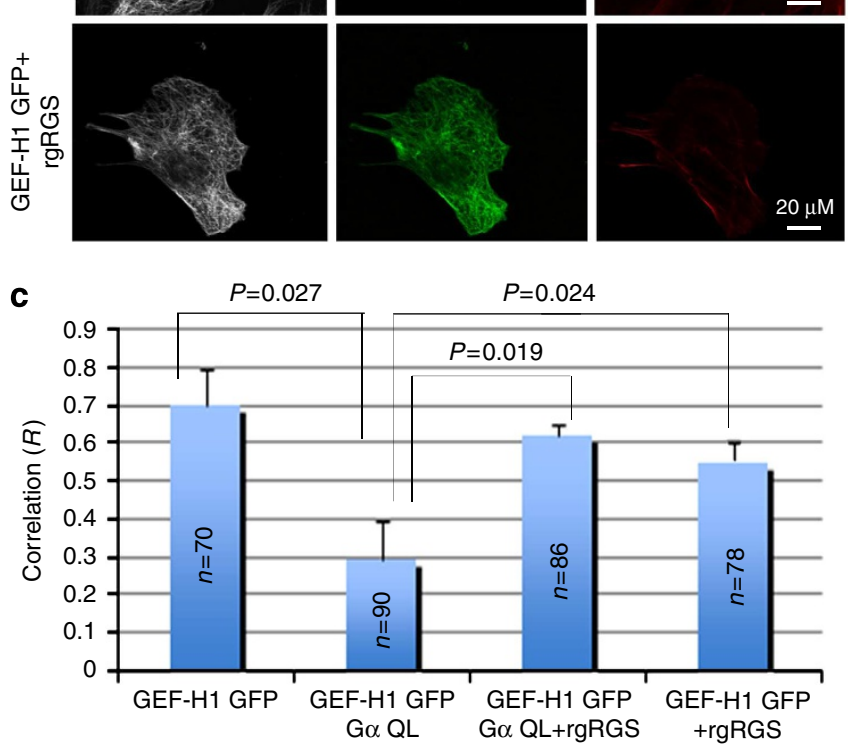

Figure 3 | $\mathbf{G} \alpha_{12}$ induces translocation and activation of GEF-H1.

(a) Induction of stress fibres by $\mathrm{G} \alpha_{12}$ requires $\mathrm{GEF}-\mathrm{H} 1$. Wild-type or $\mathrm{GEF}-\mathrm{H} 1^{-/-}$MEFs ectopically expressing $\mathrm{G} \alpha_{12}$ were stained with antiphalloidin or anti-G $\alpha_{12}$ antibodies and imaged by confocal microscopy. Scale bar, $20 \mu \mathrm{m}$. (b) $\mathrm{G} \alpha_{12}$ expression triggers GEF-H1 relocalization and activation. GEF-H1 ${ }^{-/-}$cells were transfected with GEF-H1-eGFP alone or together with a constitutively active $\mathrm{G} \alpha_{12}\left(\mathrm{G} \alpha_{\mathrm{QL}}\right)$ mutant or with $\mathrm{G} \alpha_{\mathrm{QL}}$ and dominantnegative RGS peptide (rgRGS). Cells were stained with anti-phalloidin or anti-tubulin antibodies and imaged. Scale bar, $20 \mu \mathrm{m}$. (c) The correlation coefficient of GEF-H1-eGFP and polymerized microtubules co-localization in b was determined in 70 cells (or more) from three independent experiments. Error bars represent s.d. of at least three independent replicates, and $P$ values derived from pairwise $t$-tests are indicated. 
a

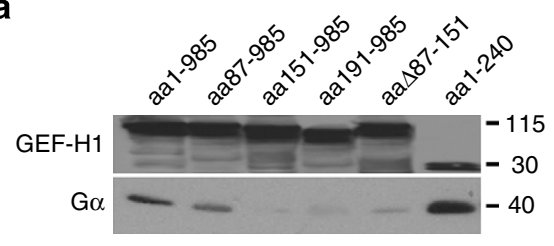

C

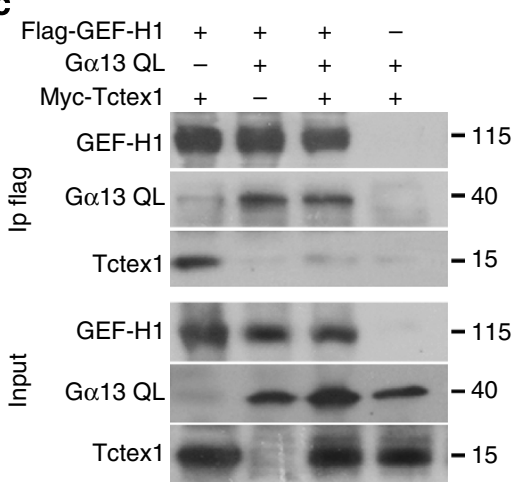

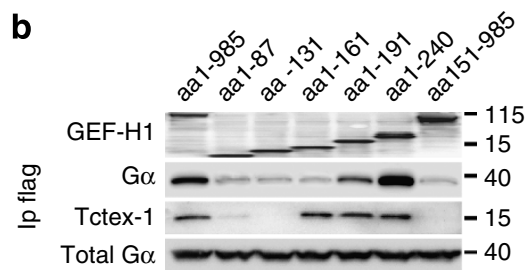

d

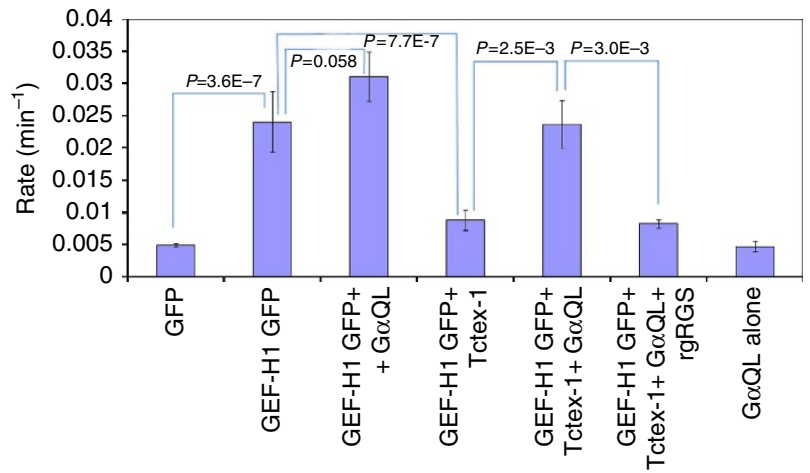

Figure 4 | The $\mathbf{G} \alpha_{\mathbf{1 2}} / \mathbf{1 3}$ subunit binds to GEF-H1, displaces Tctex-1 and stimulates exchange activity. (a) The $\mathrm{G} \alpha$-binding site resides in the $N$-terminal region of GEF-H1. His-GEF-H1 (a.a. 1-985) or a series of N- and C-terminal truncations and deletions were expressed in HEK293T cells and protein complexes were immunoprecipitated with $\alpha$-His antibodies. GEF-H1 immune complexes were immunoblotted with anti-GEF-H1 or anti-G $\alpha_{13}$ antibodies. (b) Optimal binding to endogenous $\mathrm{G \alpha}_{13}$ requires the first 240 amino acids of $\mathrm{GEF}-\mathrm{H} 1$. Flag-GEF-H1 or a series of $\mathrm{N}$-terminal fragments were expressed in HEK293T cells and protein complexes were immunoprecipitated with anti-Flag antibodies. GEF-H1 immune complexes were immunoblotted with anti-GEF-H1, anti-G $\alpha_{13}$ or anti-Tctex-1 antibodies to detect endogenous $G \alpha_{13}$ and Tctex-1, respectively. Total $G \alpha_{13}$ levels were assessed by western blot. (c) Constitutively active $\mathrm{G} \alpha_{13}$ inhibits the association of Tctex-1 with GEF-H1. Flag complexes were immunoprecipitated from HEK293T lysates expressing Flag-GEF-H1 with or without Myc-Tctex-1 and $\mathrm{G} \alpha_{13 \mathrm{QL}}$, and immunoblotted with anti-GEF-H1, anti-Tctex-1 or anti-G $\alpha_{13}$ antibodies. Western blot of GEF-H1, $\mathrm{G} \alpha_{13}$ and Tctex-1 are shown. (d) $\mathrm{G} \alpha_{13 \mathrm{QL}}$ antagonizes Tctex-1-mediated inhibition of GEF-H1 exchange activity. RhoA nucleotide exchange rates were measured in lysates from HEK293T cells expressing combinations of eGFP, GEF-H1-eGFP, Flag-Tctex-1, constitutively active G $\alpha_{130 L}$ and the inhibitory rgRGS domain, as indicated. GEF-H1, Tctex-1 and $\mathrm{G} \alpha_{\mathrm{QL}}$ expression were confirmed by western blots. The s.d. derived from at least three independent replicate experiments are shown along with $P$ values from pairwise $t$-tests.

for the entire 240-a.a. $\mathrm{N}$ terminus of GEF-H1 for $\mathrm{G} \alpha$ binding suggests either the presence of a folded domain or several multivalent-binding sites, and is consistent with the size of $\mathrm{RH}$ domains of p115, PDZ and LARG, which are 200 amino acids or greater in length.

The G $\alpha$-binding site on GEF-H1 overlaps the binding site of Tctex-1. Therefore, we asked whether $\mathrm{G} \alpha_{12 / 13}$ competes with Tctex-1 binding, and whether displacement of Tctex-1 by G $\alpha_{12 / 13}$ may contribute to the activation of GEF-H1. GEF-H1 immune complexes from HEK293T cells expressing GEF-H1, Tctex-1 and $\mathrm{G} \alpha_{13 \mathrm{QL}}$ were analysed for the presence of Tctex-1 and $G \alpha_{13}$. While Tctex-1 was detected in GEF-H1 immunoprecipitates derived from cells expressing GEF-H1 and Tctex-1 alone, expression of $\mathrm{G} \alpha_{13 \mathrm{OL}}$ markedly reduced the association of Tctex-1 with GEF-H1 (Fig. 4c). Moreover, G $\alpha_{13 \mathrm{QL}}$ potently antagonized the inhibition of GEF-H1 exchange activity by overexpressed Tctex-1 in an rgRGS-sensitive manner (Fig. 4d), consistent with the observation that $\mathrm{G} \alpha_{13 \mathrm{QL}}$ functionally competes with Tctex-1. In the absence of Tctex-1 overexpression, GEF-H1 exchange activity increased when $\mathrm{G} \alpha_{13 \mathrm{QL}}$ was coexpressed, however, this trend did not reach statistical significance, supporting the hypothesis that displacement of Tctex-1 is the major mechanism by which $\mathrm{G} \alpha_{13}$ activates GEF-H1. Similar results were obtained for $\mathrm{G} \alpha_{12 \mathrm{QL}}$.

We and others have previously shown that GEF-H1 is phosphorylated on Ser885 and associated with 14-3-3 proteins in its inactive state ${ }^{3,21}$. We examined whether $\mathrm{G} \alpha_{13 \mathrm{QL}}$ disrupted the interaction of 14-3-3 with GEF-H1 as a surrogate measure of GEF-H1 activation. We probed the association of 14-3-3 with
GEF-H1 in cells with or without $\mathrm{G} \alpha_{13 \mathrm{OL}}$ expression. While 14-3-3 was detected in GEF-H1 immunoprecipitates derived from GEFH1-transfected cells, $\mathrm{G} \alpha_{13 \mathrm{QL}}$ co-expression markedly reduced the association of 14-3-3 proteins with GEF-H1 (Fig. 5a). Expression of the dominant-negative rgRGS domain blocked this effect of $\mathrm{G} \alpha_{13 \mathrm{QL}}$, and restored the interaction between 14-3-3 and GEF-H1 (Fig. 5a). We next co-expressed GEF-H1 with 14-3-3 or Tctex-1 or $\mathrm{G} \alpha_{13 \mathrm{OL}}$, immunoprecipitated 14-3-3, Tctex-1 or $\mathrm{G} \alpha_{13 \mathrm{QL}}$ and probed the status of Ser885 phosphorylation in each complex with a phospho-Ser885-specific GEF-H1 antibody. We observed that the level of GEF-H1 Ser885 phosphorylation was strongly correlated with association with 14-3-3 and Tctex-1 and inversely correlated with $\mathrm{G} \alpha_{13}$ association (Fig. 5b). These results suggest that GEF-H1 bound to $\mathrm{G} \alpha$ is in its active state (that is, dephosphorylated on Ser885).

$\mathrm{G} \alpha$ or $\mathrm{G} \beta$ is sufficient to release GEF-H1 from microtubules. Having demonstrated that either G $\beta$ or $\mathrm{G} \alpha$ can activate GEF-H1, we queried whether both subunits are required in vivo for stimulation of GEF-H1 by ligand-induced activation of GPCRs. Following stimulation with thrombin, GEF-H1 was immunoprecipitated from lysates of starved HEK293T cells expressing the rgRGS domain (to sequester $\left.G \alpha_{12 / 13}\right)^{18,19}$ and/or $\beta$ ARKct (to block $G \beta)^{16}$. The resulting immune complexes were probed for endogenous DIC as a marker of GEF-H1 localization to the microtubule-associated dynein-protein complex. While endogenous DIC was co-immunoprecipitated with Flag-GEFH1 from starved HEK293T cells (Fig. 5c), the amount of DIC 
a

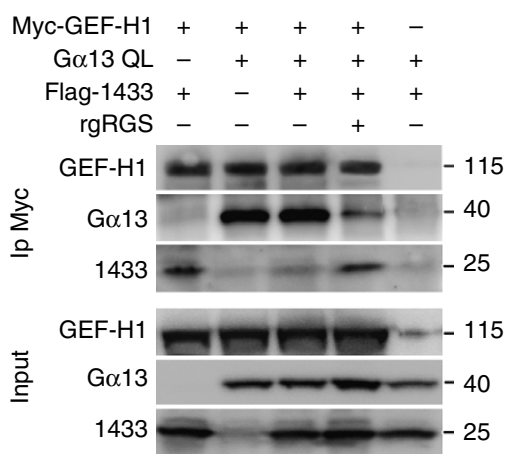

C

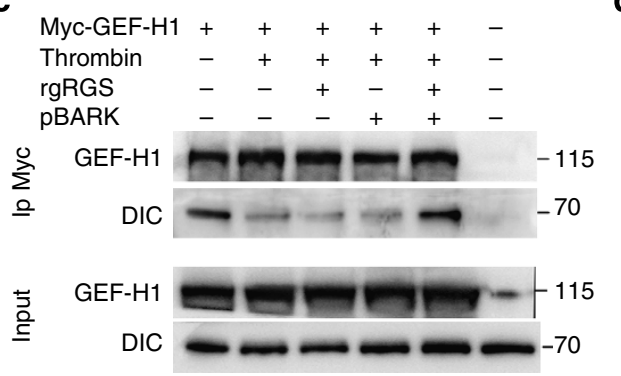

b GEF-H1 ++++

Tctex-1 +- -

1433 - + - -

Ga13QL - _ + -

Total protein

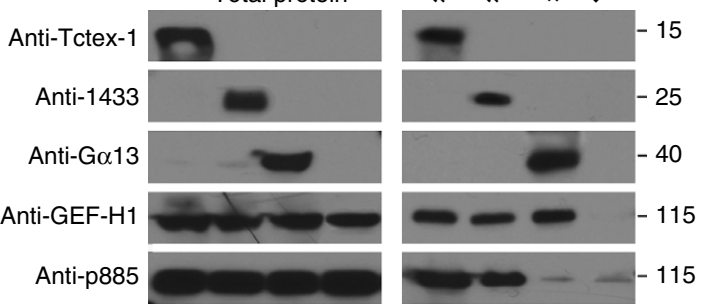

d

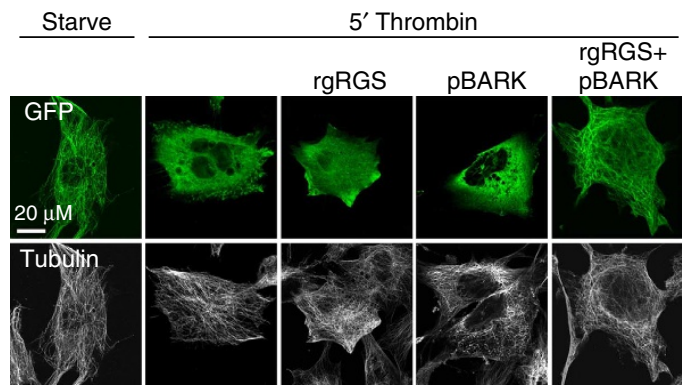

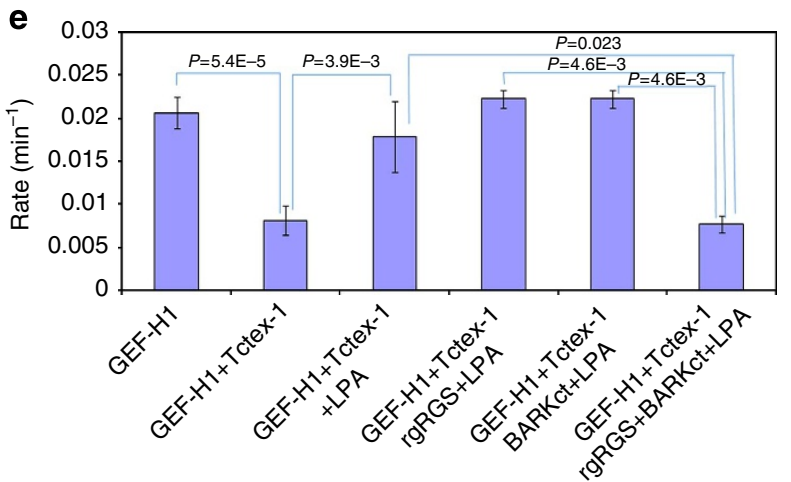

Figure 5 | $\mathbf{G} \alpha_{12 / 13}$ signalling reduces the interaction of 14-3-3 with GEF-H1 and can stimulate exchange activity independent of $\mathbf{G} \beta$. (a) $\mathrm{G} \alpha_{13 \mathrm{Q}}$ disrupts 14-3-3 protein interaction with GEF-H1. HEK293T cells were transfected with Myc-GEF-H1 with or without Flag-14-3-3 and G $\alpha_{13 Q}$, or with the dominant-negative RGS peptide (rgRGS). Myc-protein complexes were immunoblotted with anti-GEF-H1, anti-Flag or anti-G $\alpha_{13}$ antibodies. Western blots of GEF-H1, $\mathrm{G} \alpha_{13}$ and 14-3-3 in the whole-cell lysate are shown. (b) $\mathrm{G} \alpha_{13}$-bound GEF-H1 is dephosphorylated at Ser885. HEK293T cells were transfected with GEF-H1, Flag-Tctex-1, Myc-14-3-3 and $\mathrm{G} \alpha_{13 \mathrm{QL}}$. Myc- Flag- and $\mathrm{G} \alpha_{13}$ protein complexes were precipitated from lysates and immunoblotted with anti-Tctex-1, anti-14-3-3, anti-G $\alpha_{13}$, anti-GEF-H1 or anti-phosphoSer885 antibodies (right panel). Western blots of total cell lysates are shown (left). (c) Inhibition of both $\mathrm{G} \alpha$ and $\mathrm{G} \beta$ are required to inhibit thrombin-induced disruption of the GEF-H1-dynein complex. Cells were transfected with Myc-GEF$\mathrm{H} 1$ with or without rgRGS and/or $\beta$ ARKct. Cells were treated with thrombin and Myc-complexes were immunoprecipitated from lysates and blotted for endogenous GEF-H1 and DIC. Western blots of total GEF-H1 and DIC are shown below. (d) Inhibition of both G $\alpha$ and G $\beta$ are required to block thrombininduced relocalization of GEF-H1. GEF-H1 ${ }^{-/-}$cells were transfected with GEF-H1-eGFP alone or with rgRGS and/or $\beta A R K c t$. Cells were treated with thrombin, fixed and stained with anti- $\alpha$-tubulin antibodies and imaged by confocal microscopy. Scale bar, $20 \mu \mathrm{m}$. (e) Inhibition of both $\mathrm{G} \alpha$ and $\mathrm{G} \beta$ are required to block LPA-induced activation of GEF-H1 exchange activity. RhoA nucleotide exchange rates were measured in lysates from HEK293T cells expressing combinations of GEF-H1-eGFP, Tctex-1, rgRGS domain and $\beta$ ARKct peptide, with or without LPA stimulation, as indicated. The s.d. of at least three independent replicates are shown along with $P$ values from pairwise $t$-tests.

associated with GEF-H1 was markedly reduced after 5 min treatment with thrombin (Fig. 5c). Expression of either rgRGS or $\beta$ ARKct alone had little impact on thrombin's ability to disrupt the GEF-H1-DIC interaction, however, co-expression of both rgRGS and $\beta A R K c t$ blocked the disruptive effect of thrombin on the GEF-H1-DIC complex (Fig. 5c). In parallel experiments, we examined the localization of GEF-H1-eGFP following thrombin treatment in cells expressing the rgRGS domain or the $\beta$ ARKct peptide. We observed that neither the rgRGS domain nor the $\beta A R K c t$ peptide alone were sufficient to block the relocalization of GEF-H1 by thrombin, however, the expression of both inhibitory proteins had a potent blocking effect on GEF-H1 relocalization (Fig. 5d). These results provide evidence that either $\mathrm{G} \alpha$ or $\mathrm{G} \beta$ is sufficient to trigger the displacement of GEF-H1 from the microtubules. Finally, we used the rgRGS domain and/ or the $\beta$ ARKct peptide to examine whether $G \beta$ and $G \alpha$ exhibit functionally redundant or cooperative roles in the GPCRstimulated activation of GEF-H1. The inhibitory effect of Tctex1 on GEF-H1 exchange activity was relieved by treatment of cells with LPA, and this was not impacted by expression of rgRGS or $\beta A R K c t$ alone (Fig. 5e). However co-expression of rgRGS and $\beta A R K c t$ together blocked the ability of LPA to relieve Tctex-1 
inhibition of GEF-H1, further suggesting that either $\mathrm{G} \alpha$ or $\mathrm{G} \beta$ is sufficient to induce GEF-H1 activation.

GEF-H1 associates with and is dephosphorylated by PP2A. A clue as to the identity of the phosphatase that dephosphorylates GEF-H1 Ser885 was obtained from a systematic survey of all PP2A catalytic, scaffolding and regulatory subunit interactions. In this screen, Flag-tagged components of the PP2A holoenzyme were expressed in 293 Flp-In T-REx cells, and used for affinity purification coupled to mass spectrometry. GEF-H1 was detected with several of the bait proteins in this screen (Supplementary Table I). Spectral counts were extracted for GEF-H1 and normalized to spectral counts for the PP2Ascaffolding A subunit (PPP2R1A) to control for differences in expression of the different regulatory subunits. While GEF-H1 was readily recovered with both the catalytic and the scaffolding subunits, it only co-purified with members of one of the regulatory (B) families, the $\mathrm{B}^{\prime}$ regulatory subunits PPP2R5A, PPP2R5B and PPP2R5E (Fig. 6a), suggesting specificity in the regulatory subunit selection. We validated the interactions between GEF-H1 and the PP2A subunits by co-immunoprecipitation and showed that endogenous GEF-H1 and endogenous PP2A catalytic subunit proteins bind to one another (Fig. 6b). Next, we confirmed the specificity in regulatory subunit selection by co-immunoprecipitation followed by immunoblotting using the stable cell lines used in Fig. 6a: Flag-tagged PP2A catalytic subunit and $\mathrm{B}^{\prime}$ regulatory subunit, but not $\mathrm{B}, \mathrm{B}^{\prime \prime}$ or $\mathrm{B}^{\prime \prime \prime}$ subunits, each precipitated endogenous GEF-H1 (Fig. 6c). To map the regions of GEF-H1 involved in PP2A binding, we expressed truncated variants of GEF-H1 and probed for the catalytic subunit of PP2A in GEF-H1 immune complexes (Fig. 6d). The endogenous PP2Ac subunit was present in precipitates of full-length GEF-H1, the Dbl-homology (DH) and pleckstrin-homology $(\mathrm{PH})$ domain (236-572) and the $\mathrm{PH}$ domain (473-572), but not in complexes formed by the $\mathrm{DH}$ domain alone, thus localizing the PP2Ac-binding site to the $\mathrm{PH}$ domain of GEF-H1 (Fig. 6d).

We sought to determine whether PP2A was the phosphatase responsible for the dephosphorylation required for GEF-H1 activation. Flag-GEF-H1 derived from starved HEK293T cells was highly phosphorylated, as determined by an antibody recognizing the phosphorylated 14-3-3-binding motif (Fig. 7a). Immunopurified Flag-GEF-H1 was then incubated with $0.1 \mathrm{U}$ of recombinant PP2A catalytic subunit or buffer control, and a time course of the phosphorylation state was followed by western blot using

a

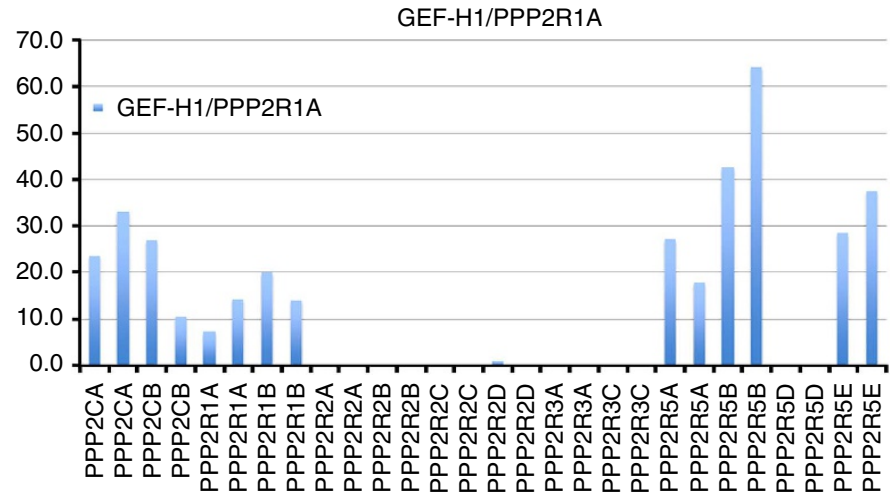

b

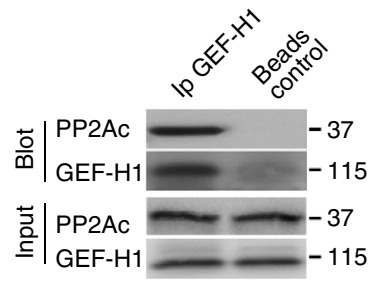

C

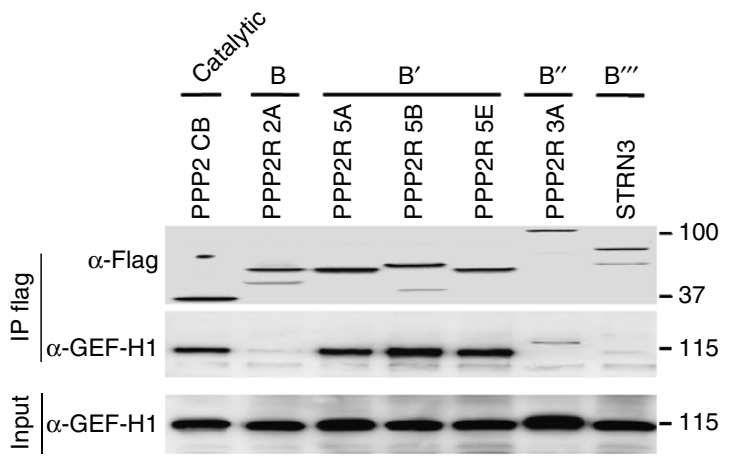

d

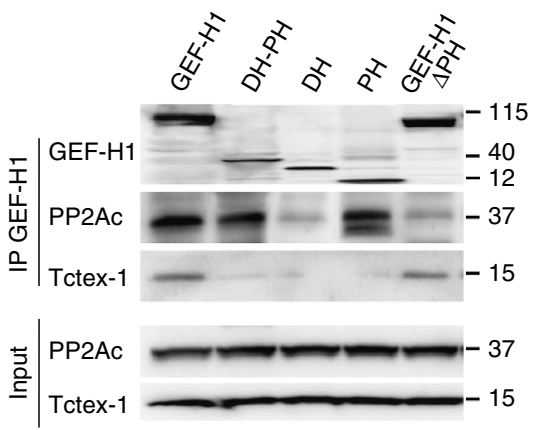

Figure 6 | The interaction of PP2A with GEF-H1 is inducible. (a) Identification of GEF-H1 as an interaction partner with specific PP2A subunits. Spectral counts from affinity purification and mass spectrometry (AP-MS) experiments for GEF-H1 and the A subunit PPP1R1A using the catalytic (PPP2CA, PPP2CB), scaffolding (PPP2R1A, PPP2R1B) and indicated regulatory B subunits as bait. Baits are represented on the $x$ axis with two biological replicates performed per bait. The $y$ axis indicates GEF-H1 spectra normalized to PPP2R1A (number spectra for GEF-H1/number spectra for PPP2R1A) $\times 100$. Values were plotted independently for each replicate to assess the reproducibility of the measurements. (b) Endogenous GEF-H1 and endogenous catalytic subunit of PP2A interact. Wild-type MEF cells growing in DMEM 10\% FBS were lysed and GEF-H1 immune complexes were probed for endogenous PP2A catalytic subunit with anti-PP2Ac antibodies. Total GEF-H1 and PP2Ac expression levels are shown. (c) GEF-H1 interacts with PP2A catalytic and B' subunits. Flp-In T-RexHEK293 cells, each stably expressing a distinct Flag-PP2A subunit, were lysed and PP2A subunits were immunoprecipitated with anti-Flag antibodies (row 1). PP2A subunit complexes were probed for endogenous GEF-H1 using anti-GEF-H1 antibodies (row 2). (d) PP2A binds to the pleckstrin-homology $\mathrm{PH}$ ) domain of GEF-H1. Flag-tagged full-length and truncated variants of GEF-H1 (DH, Dbl-homology; PH, pleckstrin-homology) were precipitated using anti-Flag antibodies (row 1). These complexes were probed for the presence of the endogenous catalytic subunit of PP2A using anti-PP2Ac antibodies (row 2). GEF-H1 and PP2Ac levels in cell lysates are shown (rows 3 and 4). 

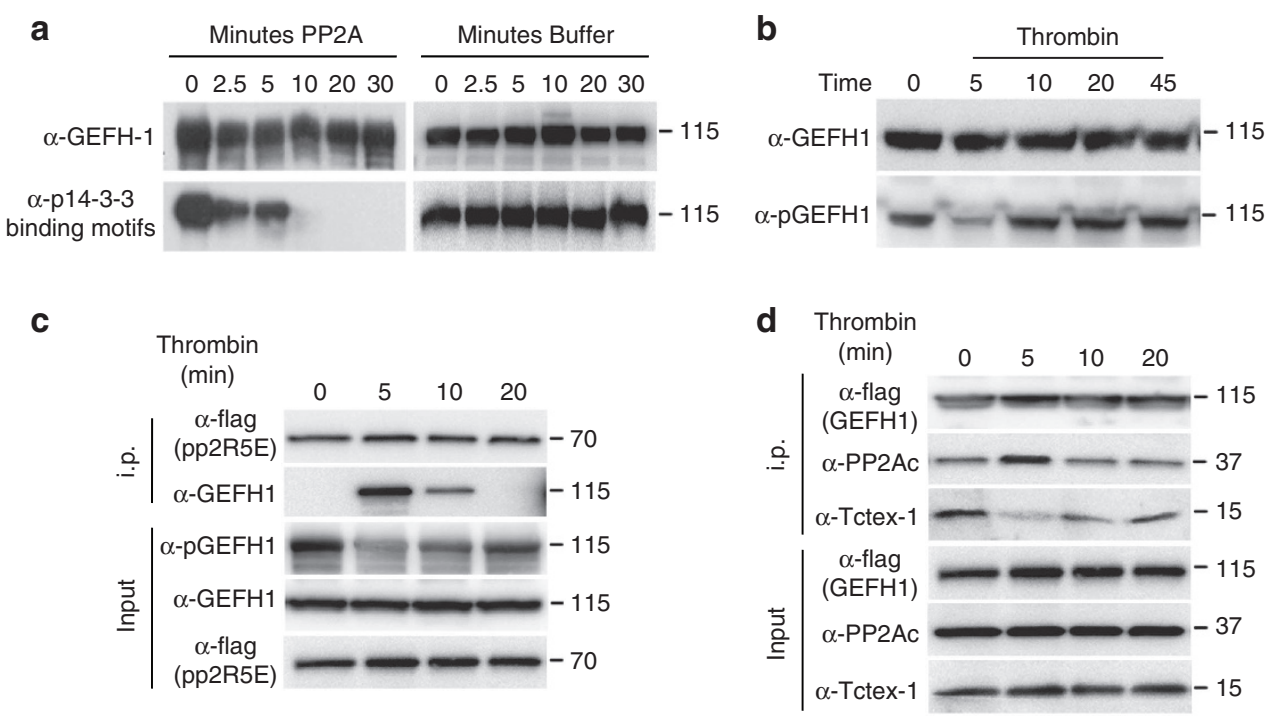

Figure 7 | PP2A dephosphorylates GEF-H1 at 14-3-3-binding sites in response to thrombin. (a) PP2A dephosphorylates GEF-H1 in vitro. Cells expressing Flag-GEF-H1 were treated with calyculin, GEF-H1 immune complexes were immunoprecipitated and incubated with recombinant catalytic subunit of PP2A for different time periods. GEF-H1 immunocomplexes were probed for GEF-H1 and for phosphorylated 14-3-3-binding motifs using anti-Flag or antiphospho14-3-3 recognition site antibodies, respectively. (b) Thrombin treatment induces rapid but transient dephosphorylation of GEF-H1 at Ser885. Cells were treated with thrombin and lysates probed with an anti-GEF-H1 phosphoSer885 antibody. (c,d) Thrombin treatment induces association between PP2A and GEF-H1. (c) Cells stably expressing Flag-PPP2R5E were treated with thrombin for different times. Flag-PPP2R5E immune complexes were probed for the presence of endogenous GEF-H1. Lysates were probed for total levels of Flag-PPP2R5E, GEF-H1 and phosphoS885. (d) Cells expressing Flag-GEF-H1 were treated with thrombin for different times. Flag-GEF-H1 immune complexes were isolated and probed for the presence of the endogenous catalytic subunit of PP2A and for endogenous Tctex-1. Lysates were probed for total levels of Flag-GEF-H1, PP2Ac and Tctex-1.

phospho 14-3-3-binding motif recognition antibody. Dephosphorylation of GEF-H1 at the 14-3-3 consensus sites by recombinant PP2Ac occurred within $10 \mathrm{~min}$ (Fig. 7a), demonstrating that GEF-H1 can serve as a PP2A substrate. Together, these data demonstrate that GEF-H1 physically interacts with PP2A, which can dephosphorylate 14-3-3-binding sites in vitro. Since the inhibited state of GEF-H1 is associated with phosphorylation of the driver 14-3-3 site Ser885, and mutation of this site (Ser885A) causes constitutive activation of GEF-H1 (ref. 6), we wanted to determine whether thrombin treatment specifically induced dephosphorylation of Ser885. Starved cells were treated with thrombin and cells were lysed over a time course of $45 \mathrm{~min}$, and immunoblotted with a phospho-Ser885specific GEF-H1 antibody. Ser885 phosphorylation was detected in starved cells, but decreased after $5 \mathrm{~min}$ of thrombin treatment (Fig. 7b). GEF-H1 dephosphorylation at Ser885 was transient, with the return of phosphorylation by $10 \mathrm{~min}$, suggesting that GEF-H1 is active for only a brief period of time.

We then examined whether the interaction between PP2A subunits and GEF-H1 increased in response to thrombin treatment, and whether the kinetics of the interaction correlated with the transient dephosphorylation of Ser885. Starved HEK293T cells expressing Flag-tagged PPP2R5E B' subunit were treated with thrombin and lysed at different time points. FlagPPP2R5E $B^{\prime}$ immunoprecipitates were probed for endogenous GEF-H1. We observed a switch-like induction of GEF-H1 association with the $\mathrm{B}^{\prime}$ subunit at $5 \mathrm{~min}$ following thrombin treatment (Fig. 7c). This interaction was reduced at $10 \mathrm{~min}$ and was undetectable after $20 \mathrm{~min}$ of thrombin treatment (Fig. 7c). The GEF-H1:PP2A interaction was anti-correlated with Ser885 phosphorylation (Fig. $7 \mathrm{c}$, third panel), with the protein complex assembling to its maximum at $5 \mathrm{~min}$ following thrombin treatment and decreasing towards baseline thereafter. We observed similar kinetics in the interaction between GEF-H1 and the endogenous PP2A catalytic subunit following thrombin treatment (Fig. 7d). Importantly, the levels of GEF-H1 associated with the microtubule dynein complex, as measured by the association of GEF-H1 with Tctex-1, decreased in response to thrombin (Fig. 7d). These results show that PP2A and GEF-H1 form a dynamic complex in response to thrombin, which is temporally correlated to dephosphorylation of the GEF-H1 negative regulatory site Ser885, loss of dynein interaction and redistribution from the microtubule array.

Microtubule depolymerization-independent GEF-H1 activation. Our results suggest a model where GEF-H1 activation results from disruption of the GEF-H1:dynein complex by G-protein subunits in the absence of microtubule depolymerization ${ }^{6,7}$. To test this model, we next determined whether Taxol-induced stabilization of microtubules could inhibit LPA or thrombin-mediated activation of GEF-H1. We measured the effect of Taxol on GPCR-mediated GEF-H1 dephosphorylation, stress fibre formation and GEF activation. Starved HEK293T cells were pre-treated with Taxol and then stimulated with thrombin. Lysates were recovered at different time intervals and probed for the levels of GEF-H1 pSer885. We found that Taxol did not affect the kinetics or the magnitude of thrombin-induced GEF-H1 dephosphorylation compared with untreated control cells (Fig. 8a). Similarly, we examined whether Taxol could suppress LPA or thrombininduced GEF-H1 redistribution from the microtubule array and stress fibre formation. GEF-H1 knockout MEFs were transfected with GEF-H1-eGFP, starved for 3 days, exposed to Taxol and visualized by confocal microscopy after LPA or thrombin treatment. Pretreatment of cells with Taxol failed to suppress LPA or thrombin-stimulated GEF-H1 redistribution from the microtubule to the cytoplasm and the formation of stress fibres (Fig. 8b,d). To ensure that the microtubules were not undergoing 


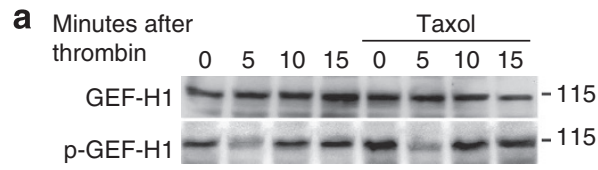

b

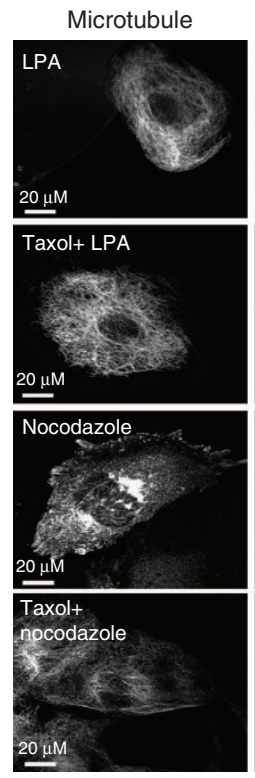

GEF-H1 GFP
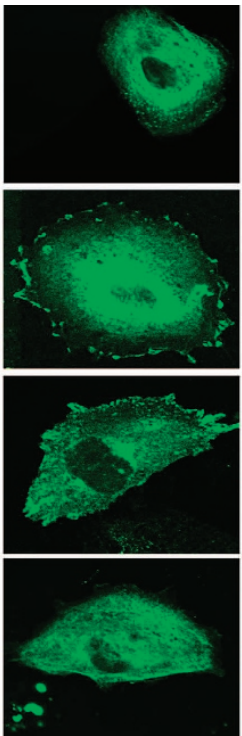

Phalloidin
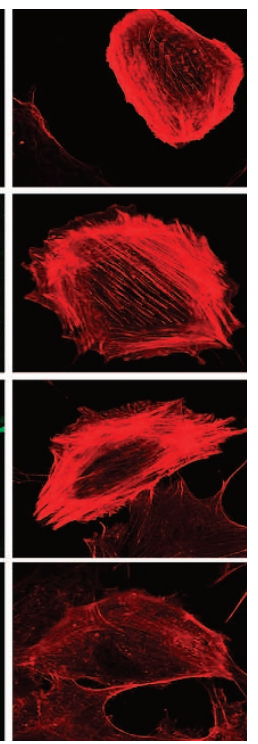

C

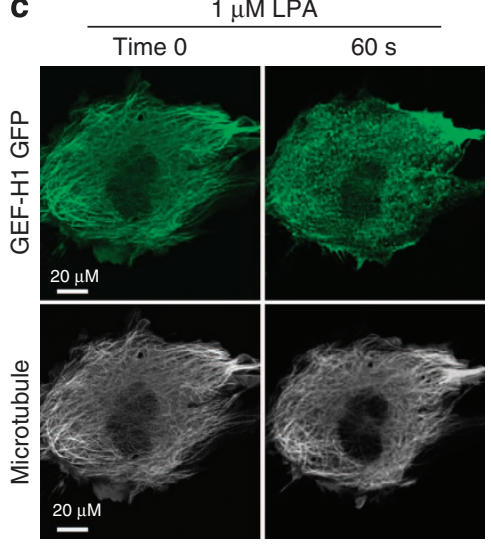

d

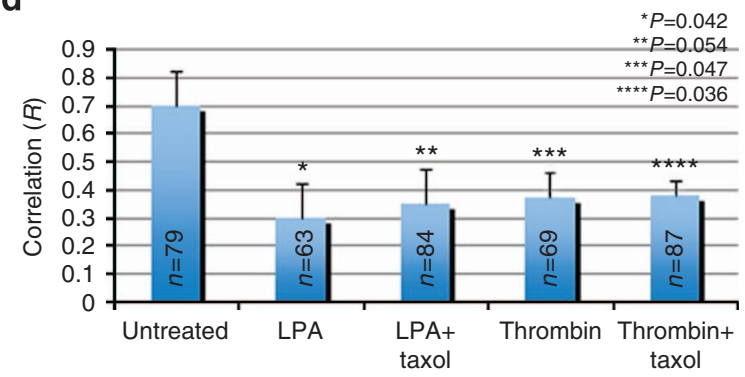

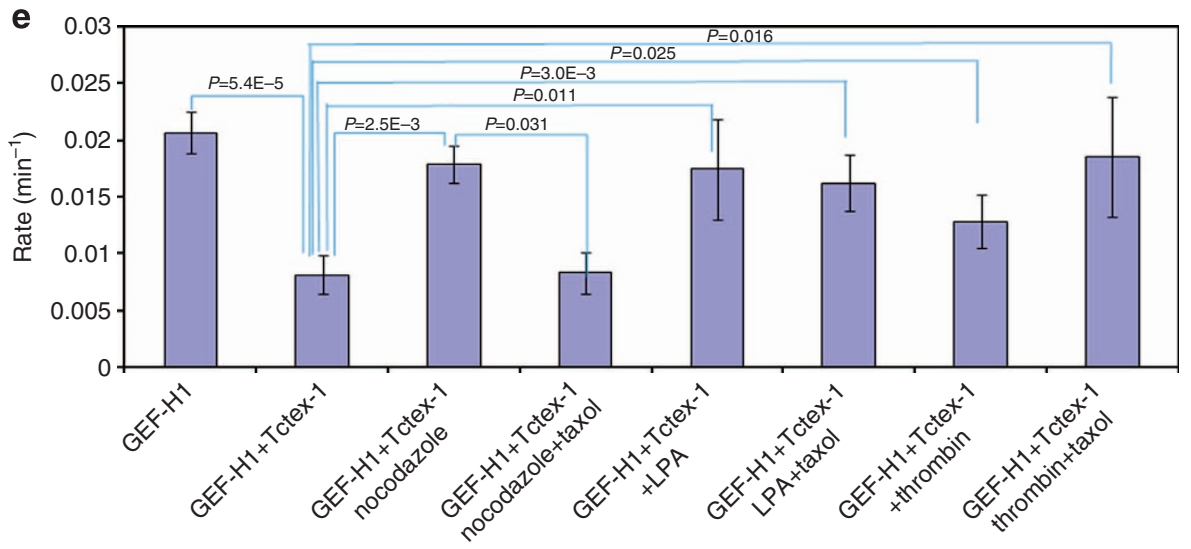

Figure 8 | GPCR-mediated activation of GEF-H1 does not require microtubule depolymerization. (a) Thrombin-induced GEF-H1 dephosphorylation is independent of microtubule depolymerization. Cells were treated with thrombin for different times with or without Taxol pretreatment. Lysates were probed for GEF-H1 and phosphoSer885 GEF-H1 levels by western blot. (b) Taxol suppresses nocodazole but not LPA-stimulated redistribution of GEF-H1 from the microtubule to the cytoplasm. GEF-H1-/- MEFs were transfected with GEF-H1-eGFP, starved for 3 days and then treated with LPA, thrombin or nocodazole with or without 24-h Taxol pretreatment. Cells were fixed and stained with anti-phalloidin and anti-tubulin antibodies and imaged by confocal microscopy. Scale bar, $20 \mu \mathrm{m}$. (c) LPA-induced GEF-H1 relocalization is independent of microtubule depolymerization. Real-time live-cell images of GEF-H1 and microtubules following LPA stimulation. GEF-H1 - / - MEFs were transiently transfected with GEF-H1-eGFP and Cherry tubulin, treated by Taxol then stimulated with LPA. Scale bar, $20 \mu \mathrm{m}$. (d) The correlation coefficient $(R)$ measuring co-localization between GFP (GEF-H1) and polymerized microtubules in $\mathbf{b}$ was determined in at least 63 cells from three independent experiments. Error bars represent s.d. of at least three independent replicates, and $P$ values derived from pairwise $t$-tests are indicated. (e) Taxol suppresses nocodazole-but not LPA-or thrombin-induced activation of GEF-H1 exchange activity. RhoA nucleotide exchange rates were measured in the lysates derived from HEK293T cells overexpressing GEF-H1-eGFP or co-expressing GEF-H1-eGFP and Flag-Tctex-1, untreated or treated with LPA, thrombin or $20 \mu \mathrm{M}$ nocodazole, with or without 24-h pretreatment with Taxol. The s.d. derived from at least three independent replicates are indicated, with $P$ values from pairwise $t$-tests.

rapid cycles of depolymerization/repolymerization, even in the presence of Taxol, we expressed GEF-H1-eGFP together with cherry tubulin, treated the cells with Taxol and visualized them by real-time confocal microscopy. GEF-H1 rapidly relocalized into a cytoplasmic vesicular pattern within $60 \mathrm{~s}$ of LPA stimulation, in the absence of microtubule depolymerization (Fig. 8c). Moreover, 
the microtubule stabilizing effect of Taxol could not antagonize activation of GEF-H1 by LPA or thrombin (Fig. 8e; Supplementary Fig. 5). These data provide evidence that GPCRmediated activation of GEF-H1 does not require disassembly of the microtubule array. Since the microtubule-depolymerizing agent nocodazole activates GEF-H1 by direct microtubule depolarization (Fig. 8b, third row and Fig. 8e), we investigated whether pretreatment of cells with Taxol could suppress this effect. We observed that pretreatment with Taxol prior to exposure to nocodazole blocked the ability of nocodazole to stimulate both stress fibre formation (Fig. 8b) and GEF-H1 activation (Fig. 8e).

\section{Discussion}

We report here a multistep mechanism for GPCR-mediated activation of the RhoGEF GEF-H1. We show that both G $\alpha$ and G $\beta \gamma$ subunits operate in a concerted manner to activate GEF-H1 through its displacement from the microtubule array, where it is anchored in an inhibited state by the dynein motor light-chain Tctex-1. In addition, we have discovered that following thrombin stimulation, the PP2A phosphatase associates with GEF-H1 and dephosphorylates the negative regulatory 14-3-3-binding site, Ser885.

RhoGEFs are activated by G-protein subunits through a variety of different mechanisms ${ }^{13}$. For example, $\mathrm{G} \alpha_{12 / 13}$ subunits activate p115-RhoGEF, LARG and PDZ-RhoGEF ${ }^{18}, \mathrm{G} \alpha \mathrm{q} / 11$ activates p63RhoGEF and TrioC, whereas G $\beta \gamma$ can directly bind and activate P-Rex1 and P-Rex2, p114RhoGEF and Arhgef5 (refs 13,22).

$\mathrm{G} \alpha_{12 / 13}$ subunits bind to their target proteins through a conserved domain called the RGS. There are over 39 mammalian RGS- and RH-domain-containing proteins ranging from small proteins comprised solely of an RGS domain to multi-domain proteins, which serve as scaffolds to diversify downstream signalling events. The canonical RGS domain consists of a nine- $\alpha$-helix bundle, which in some instances can stabilize the $\mathrm{G} \alpha_{12 / 13}$ transition state required to accelerate the hydrolysis of GTP, which inactivates $G \alpha$ thus controlling the longevity of G-protein signalling ${ }^{23-26}$. The RhoGEFs p115-RhoGEF, LARG and PDZ-RhoGEF bind to $\mathrm{G} \alpha_{12 / 13}$ subunits through a $\mathrm{RH}$ domain $^{27}$ contained in their peptide sequence. The $\mathrm{RH}$ domains of the p115RhoGEF ${ }^{28}$ and the PDZ-RhoGEF ${ }^{29}$ share a conserved core with the RGS domain, but possess additional $\mathrm{N}$ - and $\mathrm{C}$-terminal extensions that mediate a distinct mode of interaction with $\mathrm{G} \alpha . \mathrm{G} \alpha_{12 / 13}$ localizes GEFs to the plasma membrane or stimulates GEF activity directly through the release of an autoinhibitory domain or both ${ }^{18,30-33}$

GEF-H1 lacks a canonical RGS domain, nevertheless, in this study, we have shown that active $\mathrm{G} \alpha_{12 / 13}$ binds to a region encompassing the first 240 amino acids of GEF-H1 and stimulates its intrinsic exchange activity. We carefully examined whether this region of GEF-H1 shares any structural features of a $\mathrm{RH}$ domain, however, the $\mathrm{N}$ terminus of GEF-H1 cannot be aligned with $\mathrm{RH}$-containing sequences present in PDZ-RhoGEF and p115-RhoGEF. Furthermore, the DISOPRED algorithm ${ }^{34}$ predicts that this region is intrinsically disordered except for residues $40-85$, which comprise a conserved $\mathrm{C} 1$ domain predicted to form a zinc-finger-like structure. Furthermore, GEF-H1 lacks the IIG and EDF/YD/E motifs that typify the RH domains present in other RhoGEFs. Our pull-down data (Fig. 4a,b) indicated that residues 1-87 (which include the $\mathrm{C} 1$ domain) bind $\mathrm{G} \alpha$ weakly, whereas residues $87-985$ bind $\mathrm{G} \alpha$ almost as well as 1-985. These results strongly argue that $\mathrm{G} \alpha$ binding to GEF-H1 involves an intrinsically disordered region in the N-terminal portion of GEF$\mathrm{H} 1$, which may undergo binding-coupled folding. Such proteinprotein interactions involving intrinsically disordered regions have been seen in many signalling proteins and transcription factors $^{35}$. More comprehensive understanding of this unique mode of $\mathrm{G} \alpha$ interaction awaits further structural studies to elucidate the detailed binding interface of GEF-H1 and $\mathrm{G} \alpha_{12 / 13}$.

We have demonstrated that the consequences of $\mathrm{G} \alpha$ binding to GEF-H1 include displacement of GEF-H1 from the microtubule array. This effect is most likely a direct result of competitive binding between $\mathrm{G} \alpha_{12 / 13}$ and Tctex-1, since they share an overlapping binding site within the $\mathrm{N}$ terminus of GEF-H1. $\mathrm{G} \alpha_{12 / 13}$ activates GEF-H1 first by displacing GEF-H1 from the microtubule-associated inhibited sink, however, it is possible that $\mathrm{G} \alpha_{12 / 13}$ binding to the $\mathrm{N}$ terminus of GEF-H1 might also relieve a potential negative regulatory mechanism in a manner similar to the regulation of the $\mathrm{RH}$-family of GEFs.

In addition to the action of $\mathrm{G} \alpha$, we have shown $\mathrm{G} \beta \gamma$ subunits activate GEF-H1 by targeting the Tctex-1:DIC-binding interface. Tctex- 1 was found to bind to $G \beta \gamma$ subunits in a functional genetic screen looking for receptor-independent activators of G-proteins called activator of G-protein signalling ${ }^{14}$. In a subsequent study, Sachdev et al. ${ }^{15}$ found that $G \beta \gamma$ binds to a dynein-free cytoplasmic fraction of Tctex-1. They showed that G $\beta$ competes with the DIC to displace Tctex-1 from the dynein motor complex. Our data demonstrate that G $\beta \gamma$ subunits operating downstream of thrombin or LPA receptors dispace and activate GEF-H1 through a similar mechanism. We propose that free $G \beta \gamma$ subunits bind to Tctex-1, resulting in the displacement of Tctex-1 and GEF-H1 from DIC and the dynein complex. Therefore, both G $\alpha$ and G $\beta \gamma$ collaborate to disrupt the GEF-H1:Tctex-1:DIC inhibited complex by competing with the GEF-H1:Tctex-1 and Tctex-1:DIC interfaces, respectively. To our knowledge, this is the first report of a system in which both the $G \alpha$ and $G \beta$ subunits signal to activate a pathway by engaging distinct components of the same complex.

Nocodozole-induced microtubule depolymerization potently activates GEF-H1 (refs 5-7), thus GEF-H1 activity during mitosis may be a consequence of the change in microtubule dynamics during the reorganization of stable interphase microtubules into the mitotic spindle ${ }^{36}$. Our data demonstrate that GEF-H1 can be activated in interphase through a second mechanism that is independent of microtubule disassembly. We show that LPA or thrombin treatment leads to the acute relocalization of GEFH1 off the microtubule array without evidence of microtubule disruption. Whereas Taxol suppressed the effects of nocodazolemediated microtubule depolymerization and GEF-H1 activation, it had no effect on inhibiting LPA- or thrombin-mediated microtubule relocalization or activation of GEF-H1. Therefore, disruption of the GEF-H1:Tctex-1-inhibited complex following LPA treatment by the concerted actions of $G \alpha$ and $G \beta \gamma$ are sufficient to activate GEF-H1 in the absence of microtubule depolymerization. A similar phenomenon has recently been reported where the activation of GEF-H1 following the application of tensional force on cells was independent of microtubule depolymerization ${ }^{10}$. Thrombin-induced partial depolymerization of microtubules has been described in endothelial cells ${ }^{2}$. We observed such partial depolymerization of microtubules in response to thrombin treatment only at time points after GEF-H1 had already been released from the microtubules. Moreover, blocking this depolymerization by Taxol did not prevent GEF-H1 relocalization. Interestingly, GEF-H1 displacement from microtubules may contribute to microtubule depolymerization in response to thrombin, as GEF$\mathrm{H} 1$ has been reported to promote microtubule stability ${ }^{37}$.

GEF-H1's activity in normal cells is tightly regulated through phosphorylation $3,7,21,38$. In a previous publication, we found that PKA phosphorylates and inhibits GEF-H1 by creating a 14-3-3-binding site $^{3}$, which strongly suggests that a cellular 
phosphatase(s) is involved in the activation of GEF-H1. In this study, we provide evidence that the PP2A phosphatase positively regulates GEF-H1 by dephosphorylating Ser885. We show that GEF-H1 associates with the catalytic (C), scaffold (A) and $B^{\prime}$ regulatory subunits of the $\mathrm{PP} 2 \mathrm{~A}$ heterotrimer in cells, and that the recombinant PP2A catalytic subunit was competent to dephosphorylate the 14-3-3-binding site on GEF-H1 in vitro. Moreover, we demonstrated that activation of cells with either thrombin or LPA induced a transient GEF-H1:PP2A interaction that was temporally correlated with both dephosphorylation of Ser885 and stress fibre formation. The precise mechanisms by which G-protein subunits trigger the assembly of GEF-H1 with PP2A remain to be established.

In summary, we describe a novel multistep process by which the RhoGEF GEF-H1 is activated downstream of GPCR stimulation. We show that G-protein subunits engage in the disassembly of the GEF-H1:Tctex-1:DIC complex, which tethers GEF-H1 locked in an inhibited state to polymerized microtubules. The displacement of GEF-H1 from the microtubule array corresponds with its assembly with PP2A phosphatase subunits required for the dephosphorylation of a negative regulatory 14-33-binding site in the carboxyterminus of GEF-H1. We conjecture that the multiple molecular events required for GEF-H1 activation has evolved as a mechanism to ensure a highly restricted temporal and spatial activation of the Rho pathway downstream of GPCR ligand stimulation.

\section{Methods}

Expression constructs. Full-length GEF-H1 (accession no. AF177032) or truncated versions (as described in the figures) were cloned into the pFlag-CMV2 vector (Sigma). Complementary DNAs for all full-length and truncated versions of GEF-H1 and $\Delta 87-151$ GEF-H1 were cloned into pcDNA3.1-His/Myc vector (Invitrogen). eGFP-tagged GEF-H1 and Tctex-1 were constructed by cloning fulllength GEF-H1 or Tctex-1 (accession no. NM_174620) into peGFP-C1 (Invitrogen). GEF-H1, $\Delta 151$ GEF-H1 and Tctex-1 were cloned into the BglII/AgeI and BglII/EagI sites of pCMV-HA-VC155 and pCMV-HAVN173, respectively.

Cell culture and transfection. MEFs derived from GEF-H1 $1^{-/-}$(ref. 6) or wildtype littermate embryos and HEK293T cells were cultured in Dulbecco's modified Eagle's medium (DMEM, Life Technologies Inc.) supplemented with 10\% fetal bovine serum (FBS) (HyClone). MEFs and Rat2 cells were transfected using Effectene (QIAGEN), and HEK293T using Polyfect, according to the manufacturer's instructions. All cultures were maintained in a $5 \% \mathrm{CO}_{2}$ environment at $37^{\circ} \mathrm{C}$. For cell spreading and attachment analyses, cells were propagated at low passage number in the presence of DMEM with $10 \%$ FBS.

\begin{abstract}
Antibodies. Rabbit anti-DYNLT1 antibody (anti-Tctex-1) was purchased from Proteintec (11954-1-ap). Polyclonal sheep anti-GEF-H1 antibodies were described previously ${ }^{36}$. Mouse anti-vinculin antibody (anti-vinculin) was purchased from Sigma (V9131), anti-14-3-3 from Santa Cruz (sc-1657), anti-PP2A catalytic alpha from Becton Dickinson (05-421) and anti-phospho-Ser 14-3-3-binding motif from Cell Signaling Technology (4E2, 9606). Rabbit anti-phospho-S885 antibody was from Cell Signaling Technology. Mouse monoclonal antibody directed against bovine $\alpha$-tubulin (236-10501, A-11126), Pacific Blue anti-mouse IgG (P31582), Texas Red anti-rabbit IgG (T-2767), Texas Red anti-mouse IgG (T-862), Texas Red phalloidin (T7471), Alexa Fluor 350 phalloidin (A22281) and Alexa Fluor 594 donkey anti-sheep IgG (A-11016) were obtained from Invitrogen. Western blotting and immunofluorescence was performed using the following primary antibodies; anti-Flag (M2, F3165, Sigma), anti-His (H15, sc-803), anti-GST (B-14, sc-138), anti-Myc (Sigma, M4439), anti-PP2Ac (Millipore, 05-421) and anti-HA (H6908, Sigma). HRP-conjugated anti-mouse or anti-rabbit secondary antibodies were from GE Healthcare. Supplementary Figs 7-12 show full scans of the western blots presented in the main figures.
\end{abstract}

Immunofluorescence imaging. Cells grown on glass coverslips were treated as indicated in the corresponding figure legends and fixed with $4 \%$ paraformaldehyde for $10 \mathrm{~min}$, washed three times with phosphate-buffered saline (PBS) and permeabilized with $0.1 \%$ Triton-X-100 for $5 \mathrm{~min}$. The coverslips were blocked with $0.5 \% \mathrm{w} / \mathrm{v}$ bovine serum albumin (BSA) in PBS for $1 \mathrm{~h}$ at room temperature and incubated with primary antibody (anti-DYNLT1 1:400, sheep anti-GEF-H1 1:150, anti-tubulin 1:300 and anti-vinculin 1:400) or Texas Red phalloidin (1:400) in $0.5 \%$ $\mathrm{BSA} / \mathrm{PBS}$ at $37^{\circ} \mathrm{C}$ for $30 \mathrm{~min}$ or at $4^{\circ} \mathrm{C}$ overnight. Coverslips were washed three times with PBS and incubated with secondary antibody $(1: 500)$ at $37^{\circ} \mathrm{C}$ for $1 \mathrm{~h}$. For double labelling, slides were stained sequentially with primary and secondary antibodies at $37^{\circ} \mathrm{C}$ for $30 \mathrm{~min}$, followed by $4^{\prime}, 6$-diamidino-2-phenylindole (Molecular Probes, Invitrogen) to stain nuclei. Slides were mounted using GelTol mounting medium (Shandon Immunon, Thermo Electron Corporation). Confocal imaging was performed with an Olympus IX81 inverted microscope using a $\times 60$ zoom $\times$ 3(1.4 NA; PlanApo, Nikon) objective, and FluoView software (Olympus, Tokyo, Japan). Resolution was $512 \times 512$ with 12 bits per pixel. Excitation wavelengths used for GFP, Texas Red, 4',6-diamidino-2-phenylindole or Pacific Blue were 473, 559, 358 and $405 \mathrm{~nm}$, respectively. All images in each set of experiments were acquired with the same microscope sensitivity settings. All images compared within each figure panel were acquired on the same day, with identical staining conditions, gain and contrast setting, and same magnification (except Fig. 1c). All statistical analyses were derived from 60 or more images from three independent experiments for each treatment condition.

NMR-based GEF assay. To measure GEF activity in lysates of mammalian cells, real-time NMR GEF assays were performed ${ }^{6,17}$. Nucleotide exchange assays were carried out at $20^{\circ} \mathrm{C}$ with $0.4 \mathrm{mM}{ }^{15} \mathrm{~N}$-labelled RhoA (residues 1-181) in NMR buffer (20 mM HEPES, $100 \mathrm{mM} \mathrm{NaCl}, 5 \mathrm{mM} \mathrm{MgCl}_{2}, 2 \mathrm{mM}$ Tris (2-carboxyethyl) phosphine, $10 \% \mathrm{D}_{2} \mathrm{O}, \mathrm{pH} 7.0$ ) using a Bruker $600 \mathrm{MHz}$ NMR spectrometer equipped with a $1.7-\mathrm{mm}$ microcryoprobe. Successive ${ }^{1} \mathrm{H}-{ }^{15} \mathrm{~N}-\mathrm{HSQC}$ spectra (4 scans, 10 min per spectrum) were collected and seven pairs of guanosine diphosphate (GDP)/GTP $\gamma$ S-specific peaks (V9, Q29, A56, S73, D87, T163 and W158) were used to determine the fraction of GDP-bound RhoA in each spectrum (Supplementary Fig. 6). Data were fitted to a single-phase exponential decay function to obtain the exchange rate. HEK293T cells were transfected with the indicated constructs for $48 \mathrm{~h}$, and the GEF activity in lysates was measured by harvesting cells in a minimal volume (for example, $150 \mu \mathrm{l}$ for a $6-\mathrm{cm}$ plate) of lysis buffer (1\% Triton-X, 10\% glycerol, $50 \mathrm{mM}$ HEPES pH 7.5, and Complete Protease Inhibitor cocktail (Roche)) to achieve high total protein concentration $\left(\sim 10 \mu \mathrm{g}^{-1}\right)$. All cell lysates were freshly prepared immediately before performing assays, and partially clarified by centrifugation $(16,000 \mathrm{~g}, 30 \mathrm{~s})$. The amount of GEF-H1 in each assay was standardized by eGFP fluorescence of the lysate measured by a Shimadzu RF-5301PC spectrofluorophotometer. To compare endogenous cellular GEF activity, total cellular protein in lysates was standardized using the Bradford assay. To initiate nucleotide exchange, $2 \mathrm{mM} \mathrm{GTP} \gamma \mathrm{S}$ and lysate were added to ${ }^{15} \mathrm{~N}$ RhoA-GDP. Intrinsic RhoA nucleotide exchange was measured by adding lysis buffer. Where indicated, HEK293 cells were treated prior to harvesting as follows: nocodazole $(20 \mu \mathrm{M}, 10 \mathrm{~min})$, Taxol $(10 \mu \mathrm{M})$, LPA $(1 \mu \mathrm{M}$, $30 \mathrm{~min})$ and thrombin $\left(3 \mathrm{U} \mathrm{ml}^{-1}, 1 \mathrm{~h}\right)$.

Image analysis. Image analysis for co-localization was performed with the cell profiler v2.0 1 using the 'MeasureCorrelation' module for correlation. Focal adhesion counts were performed using ImageJ v1.4q 2 (http://rsb.info.nih.gov/ij/ index.html). Vinculin-stained cell images were transformed to 16 bits and the background was subtracted using the 'background subtractor' plugin 3 . Then they were transformed into binary images and focal adhesions were identified using the find maxima tool (Noise tolerance of 20). Paired Student's $t$-tests (ref. 39) were performed to determine statistical significance between samples. Experiments were performed at least three times, and means with $P<0.05$ were considered statistically significant. The correlation coefficient $(R)$ measuring co-localization was determined from three independent experiments. Error bars represent s.d. of at least three independent replicates, and $P$ values derived from pairwise $t$-tests are indicated.

Full-length GEF-H1 expression and purification protocol. Full-length GEF-H1 with a $\mathrm{His}_{6}$ tag and an $\mathrm{N}$-terminal immunoglobulin-binding protein (BiP) secretion signal sequence was expressed in Drosophila S2 cells using the pMTTEVA vector ${ }^{6}$ S2 cells were cultured in serum-free media and induced with $6 \mu \mathrm{M} \mathrm{CdCl}_{2}$ at a density of $5 \times 10^{6}$ cells per ml for 4 days. GEF-H1 was captured with $\mathrm{Ni}^{2}+$-NTA resin and further purified by anion-exchange (HiTrap Q) and size-exclusion chromatography (Superdex 200). Purified recombinant $\mathrm{G} \alpha_{13}$ was a kind gift from P. Sternweis (UT Southwestern Medical Center).

Cell treatments. Cells were starved for 5 days in the absence of serum and treated in DMEM containing $20 \mathrm{mM}$ HEPES and $0.5 \mathrm{mg} \mathrm{ml}^{-1}$ fatty acid-free BSA (A8806, Sigma). LPA obtained from Sigma (7260) was suspended in Hank's buffered saline solution containing $0.5 \mathrm{mg} \mathrm{ml}^{-1}$ fatty acid-free BSA and $20 \mathrm{mM}$ HEPES to a stock concentration of $1 \mathrm{mM}$. Cells were treated with $1 \mu \mathrm{M} \mathrm{LPA}$ for $30 \mathrm{~min}$ or as indicated in the figures. Bovine thrombin (605157) was purchased from Calbiochem and reconstituted to a concentration of $1 \mathrm{U}^{-1}{ }^{-1}$. Cells were treated with $3 \mathrm{U} \mathrm{ml}^{-1}$ thrombin for $1 \mathrm{~h}$ or as indicated in the figures.

Proteomics analyses. Flp-In T-Rex 293 cells (Invitrogen) were stably transfected with N-terminally-FLAG-tagged PP2A constructs and protein expression was induced by tetracycline addition. FLAG-tagged proteins and their putative interaction partners were recovered by immunoprecipitation on anti-FLAG magnetic beads ${ }^{40}$. 
Mass spectroscopy. Samples digested on beads were acidified and loaded onto home-packed C18 capillary columns ( $75 \mu \mathrm{m}$ inner diameter). HPLC columns were placed in-line in front of an LTQ mass spectrometer (Thermo) equipped with a Nanosource (Proxeon) for data-dependent acquisition over a 2-h acetonitrile $2-40 \%$ gradient $^{41}$. Acquired RAW files were converted to mgf format using ProteoWizard $^{42}$, and searched with the Mascot search engine (Matrix Science, London, UK, version 2.3.02) against the human RefSeq database supplemented with the RefSeq adenovirus entries (release 45) with a precursor ion mass tolerance of 3.0 and a fragment ion mass tolerance of 0.6. Methionine oxidation and asparagine deamidation were allowed as variable modifications, and trypsin specificity (with one missed cleavage allowed) was selected. The data were analysed in the 'Analyst' module of ProHits ${ }^{43}$ and exported into Excel files for spectral normalization and manual extraction of the data corresponding to GEF-H1 and PPP2R1A (the remainder of the data set will be the topic of a separate publication). Two biological replicates analyses were performed on each of the baits. Fourteen negative control runs were also performed that consisted of cells expressing the FLAG tag fused to a green fluorescent protein. Importantly, GEF-H1 was not identified in any of the negative control runs.

Phosphatase assays. For dephosphorylation of GEF-H1 immunoprecipitates with PP2A, cells were lysed in phosphatase lysis buffer $(50 \mathrm{mM}$ Tris $\mathrm{pH} 7.5$, $150 \mathrm{mM} \mathrm{NaCl}, 1 \mathrm{mM}$ EDTA pH 8, 1\% v/v NP40, $1 \mathrm{mM}$ dithiothreitol, $1 \mathrm{mM}$ phenylsulfonylfluoride with Complete Protease Inhibitor cocktail (Roche)), and immunoprecipitates were washed twice with lysis buffer then three times with PP2A phosphatase buffer (20 mM HEPES pH 8, $1 \mathrm{mM}$ dithiothreitol and $1 \mathrm{mM}$ $\mathrm{MnCl}_{2}$ ). Beads were preheated with $100 \mu \mathrm{lP} 2 \mathrm{~A}$ phosphatase buffer for $10 \mathrm{~min}$ at $30^{\circ} \mathrm{C}$ prior to the addition of $0.1 \mathrm{U}$ PP2A (Upstate, 14-111) and incubation at $30^{\circ} \mathrm{C}$. Reactions were stopped by washing beads with lysis buffer containing phosphatase inhibitors and boiling beads for $10 \mathrm{~min}$ in $2 \times$ sample buffer prior to SDS-polyacrylamide gel electrophoresis.

Statistical analyses. Values are expressed as means \pm s.d. Paired Student's $t$-tests ${ }^{39}$ were performed to determine statistical significance between samples. Experiments were performed at least three times and means with $P<0.05$ were considered statistically significant.

\section{References}

1. Ren, Y., Li, R., Zheng, Y. \& Busch, H. Cloning and characterization of GEF-H1, a microtubule-associated guanine nucleotide exchange factor for Rac and Rho GTPases. J. Biol. Chem. 273, 34954-34960 (1998).

2. Birukova, A. A. et al. GEF-H1 is involved in agonist-induced human pulmonary endothelial barrier dysfunction. Am. J. Physiol. Lung Cell. Mol. Physiol. 290, L540-L548 (2006).

3. Meiri, D. et al. Modulation of Rho guanine exchange factor Lfc activity by protein kinase A-mediated phosphorylation. Mol. Cell. Biol. 29, 5963-5973 (2009).

4. Westwick, J. K. et al. Transforming potential of $\mathrm{Dbl}$ family proteins correlates with transcription from the cyclin D1 promoter but not with activation of Jun NH2-terminal kinase, p38/Mpk2, serum response factor, or c-Jun. J. Biol. Chem. 273, 16739-16747 (1998).

5. Krendel, M., Zenke, F. T. \& Bokoch, G. M. Nucleotide exchange factor GEF-H1 mediates cross-talk between microtubules and the actin cytoskeleton. Nat. Cell Biol. 4, 294-301 (2002).

6. Meiri, D. et al. Mechanistic insight into the microtubule and actin cytoskeleton coupling through dynein-dependent RhoGEF inhibition. Mol. Cell 45, 642-655 (2012).

7. Callow, M. G., Zozulya, S., Gishizky, M. L., Jallal, B. \& Smeal, T. PAK4 mediates morphological changes through the regulation of GEF-H1. J. Cell Sci. 118, 1861-1872 (2005).

8. Kakiashvili, E. et al. GEF-H1 mediates tumor necrosis factor-alphainduced Rho activation and myosin phosphorylation: role in the regulation of tubular paracellular permeability. J. Biol. Chem. 284, 11454-11466 (2009).

9. Kakiashvili, E. et al. The epidermal growth factor receptor mediates tumor necrosis factor-alpha-induced activation of the ERK/GEF-H1/RhoA pathway in tubular epithelium. J. Biol. Chem. 286, 9268-9279 (2011).

10. Guilluy, C. et al. The Rho GEFs LARG and GEF-H1 regulate the mechanical response to force on integrins. Nat. Cell Biol. 13, 722-727 (2011).

11. Fukuhara, S., Chikumi, H. \& Gutkind, J. S. RGS-containing RhoGEFs: the missing link between transforming $G$ proteins and Rho? Oncogene 20, 1661-1668 (2001).

12. Dutt, P., Jaffe, A. B., Merdek, K. D., Hall, A. \& Toksoz, D. Galphaz inhibits serum response factor-dependent transcription by inhibiting Rho signaling. Mol. Pharmacol. 66, 1508-1516 (2004).

13. Aittaleb, M., Boguth, C. A. \& Tesmer, J. J. Structure and function of heterotrimeric $\mathrm{G}$ protein-regulated Rho guanine nucleotide exchange factors. Mol. Pharmacol. 77, 111-125 (2010).
14. Takesono, A. et al. Receptor-independent activators of heterotrimeric G-protein signaling pathways. J. Biol. Chem. 274, 33202-33205 (1999).

15. Sachdev, P. et al. G protein beta gamma subunit interaction with the dynein light-chain component Tctex-1 regulates neurite outgrowth. Embo J. 26, 2621-2632 (2007).

16. Koch, W. J., Hawes, B. E., Inglese, J., Luttrell, L. M. \& Lefkowitz, R. J. Cellular expression of the carboxyl terminus of a $\mathrm{G}$ protein-coupled receptor kinase attenuates $\mathrm{G}$ beta gamma-mediated signaling. J. Biol. Chem. 269, 6193-6197 (1994).

17. Marshall, C. B. et al. Probing the GTPase cycle with real-time NMR: GAP and GEF activities in cell extracts. Methods 57, 473-485 (2012).

18. Hart, M. J. et al. Direct stimulation of the guanine nucleotide exchange activity of p115 RhoGEF by Galpha13. Science 280, 2112-2114 (1998).

19. Jiang, L. I., Collins, J., Davis, R., Fraser, I. D. \& Sternweis, P. C. Regulation of cAMP responses by the G12/13 pathway converges on adenylyl cyclase VII. J. Biol. Chem. 283, 23429-23439 (2008).

20. Chen, Z., Singer, W. D., Danesh, S. M., Sternweis, P. C. \& Sprang, S. R. Recognition of the activated states of Galpha13 by the rgRGS domain of PDZRhoGEF. Structure 16, 1532-1543 (2008).

21. Zenke, F. T. et al. p21-activated kinase 1 phosphorylates and regulates 14-3-3 binding to GEF-H1, a microtubule-localized Rho exchange factor. J. Biol. Chem. 279, 18392-18400 (2004).

22. Welch, H. C. et al. P-Rex1, a PtdIns $(3,4,5) \mathrm{P} 3$ - and Gbetagamma-regulated guanine-nucleotide exchange factor for Rac. Cell 108, 809-821 (2002).

23. Tesmer, J. J., Berman, D. M., Gilman, A. G. \& Sprang, S. R. Structure of RGS4 bound to AlF4--activated G(i alpha1): stabilization of the transition state for GTP hydrolysis. Cell 89, 251-261 (1997).

24. Slep, K. C. et al. Structural determinants for regulation of phosphodiesterase by a G protein at 2.0 A. Nature 409, 1071-1077 (2001).

25. Soundararajan, M. et al. Structural diversity in the RGS domain and its interaction with heterotrimeric G protein alpha-subunits. Proc. Natl Acad. Sci. USA 105, 6457-6462 (2008).

26. Tesmer, J. J. Structure and function of regulator of G protein signaling homology domains. Prog. Mol. Biol. Transl. Sci. 86, 75-113 (2009).

27. Lutz, S. et al. The guanine nucleotide exchange factor p63RhoGEF, a specific link between Gq/11-coupled receptor signaling and RhoA. J. Biol. Chem. 280, 11134-11139 (2005).

28. Chen, Z., Wells, C. D., Sternweis, P. C. \& Sprang, S. R. Structure of the rgRGS domain of p115RhoGEF. Nat. Struct. Biol. 8, 805-809 (2001).

29. Longenecker, K. L., Lewis, M. E., Chikumi, H., Gutkind, J. S. \& Derewenda, Z. S. Structure of the RGS-like domain from PDZ-RhoGEF: linking heterotrimeric g protein-coupled signaling to Rho GTPases. Structure 9, 559-569 (2001).

30. Kozasa, T. et al. p115 RhoGEF, a GTPase activating protein for Galpha12 and Galpha13. Science 280, 2109-2111 (1998).

31. Mao, J., Yuan, H., Xie, W. \& Wu, D. Guanine nucleotide exchange factor GEF115 specifically mediates activation of Rho and serum response factor by the G protein alpha subunit Galpha13. Proc. Natl Acad. Sci. USA 95, 12973-12976 (1998).

32. Fukuhara, S., Murga, C., Zohar, M., Igishi, T. \& Gutkind, J. S. A novel PDZ domain containing guanine nucleotide exchange factor links heterotrimeric $\mathrm{G}$ proteins to Rho. J. Biol. Chem. 274, 5868-5879 (1999).

33. Fukuhara, S., Chikumi, H. \& Gutkind, J. S. Leukemia-associated Rho guanine nucleotide exchange factor (LARG) links heterotrimeric $\mathrm{G}$ proteins of the G(12) family to Rho. FEBS Lett. 485, 183-188 (2000)

34. Ward, J. J., McGuffin, L. J., Bryson, K., Buxton, B. F. \& Jones, D. T. The DISOPRED server for the prediction of protein disorder. Bioinformatics 20, 2138-2139 (2004).

35. Wright, P. E. \& Dyson, H. J. Linking folding and binding. Curr. Opin. Struct. Biol. 19, 31-38 (2009).

36. Bakal, C. J. et al. The Rho GTP exchange factor Lfc promotes spindle assembly in early mitosis. Proc. Natl Acad. Sci. USA 102, 9529-9534 (2005).

37. Yoshimura, Y. \& Miki, H. Dynamic regulation of GEF-H1 localization at microtubules by Par1b/MARK2. Biochem. Biophys. Res. Commun. 408, 322-328 (2011).

38. Birkenfeld, J. et al. GEF-H1 modulates localized RhoA activation during cytokinesis under the control of mitotic kinases. Dev. Cell 12, 699-712 (2007).

39. Kirkman, T. W. Statistics to use < http://www.physics.csbsju.edu/stats/> (1996).

40. Kean, M. J., Couzens, A. L. \& Gingras, A. C. Mass spectrometry approaches to study mammalian kinase and phosphatase associated proteins. Methods 57, 400-408 (2012).

41. Goudreault, M. et al. A PP2A phosphatase high density interaction network identifies a novel striatin-interacting phosphatase and kinase complex linked to the cerebral cavernous malformation 3 (CCM3) protein. Mol. Cell. Proteomics. 8, 157-171 (2009). 
42. Kessner, D., Chambers, M., Burke, R., Agus, D. \& Mallick, P. ProteoWizard: open source software for rapid proteomics tools development. Bioinformatics 24, 2534-2536 (2008).

43. Liu, G. et al. ProHits: integrated software for mass spectrometry-based interaction proteomics. Nat. Biotechnol. 28, 1015-1017 (2010).

\section{Acknowledgements}

We thank Beatriz Gonzalez Badillo for help with cloning and cell line generation and ZhenYuan Lin for mass spectrometric analyses of the PP2A samples. We thank Paul Sternweiss for the gift of recombinant $\mathrm{G} \alpha$. Work in the Gingras lab was supported by the Canadian Institutes of Health Research (MOP-84314). A.-C.G. is the Canada Research Chair in Functional Proteomics and the Lea Reichmann Chair in Cancer Proteomics. Work in the Ikura lab was supported by a grant from Cancer Research Society. M.I. holds the Canada Research Chair in Cancer Structural Biology. The NMR facility at UHN is supported by the Canada Foundation for Innovation. This work (R.R.) was supported by the Canadian Institutes of Health Research and the Ontario Institute for Cancer Research. R.R. holds the Amgen Chair for Cancer Research at Princess Margaret Cancer Centre.

\section{Author contributions}

D. Meiri, C.B.M., D. Mokady, J.LR, and M.M. performed experiments. All authors designed the research and analyzed the data. D. Meiri, C.B.M., A.-C.G., M.I. and R.R. wrote the paper.

\section{Additional information}

Supplementary Information accompanies this paper at http://www.nature.com/ naturecommunications

Competing financial interests: The authors declare no competing financial interests.

Reprints and permission information is available online at http://npg.nature.com/ reprintsandpermissions/

How to cite this article: Meiri, D. et al. Mechanistic insight into GPCR-mediated activation of the microtubule-associated RhoA exchange factor GEF-H1. Nat. Commun 5:4857 doi: 10.1038/ncomms5857 (2014). 